\title{
Parallel algorithms for variational inclusions and fixed points with applications
}

\author{
Afrah AN Abdou ${ }^{1 *}$, Badriah AS Alamri ${ }^{1}$, Yeol Je Cho ${ }^{1,2}$, Yonghong Yao ${ }^{3}$ and Li-Jun Zhu ${ }^{4}$
}

\author{
"Correspondence: \\ Aabdou@kau.edu.sa; \\ yjcho@gnu.ac.kr \\ 'Department of Mathematics, King \\ Abdulaziz University, Jeddah, 21589 \\ Saudi Arabia \\ Full list of author information is \\ available at the end of the article
}

\begin{abstract}
In this paper, we introduce two parallel algorithms for finding a zero of the sum of two monotone operators and a fixed point of a nonexpansive mapping in Hilbert spaces and prove some strong convergence theorems of the proposed algorithms. As special cases, we can approach the minimum-norm common element of the zero of the sum of two monotone operators and the fixed point of a nonexpansive mapping without using the metric projection. Further, we give some applications of our main results.
\end{abstract} MSC: 49J40; 47J20; 47H09; 65J15

Keywords: monotone operator; nonexpansive mapping; zero point; fixed point; resolvent

\section{Introduction}

Let $H$ be a real Hilbert space. Let $A: H \rightarrow H$ be a single-valued nonlinear mapping and $B: H \rightarrow 2^{H}$ be a set-valued mapping.

Now, we are concerned with the following variational inclusion:

Find a zero $x \in H$ of the sum of two monotone operators $A$ and $B$ such that

$$
0 \in A x+B x
$$

where 0 is the zero vector in $H$.

The set of solutions of the problem (1.1) is denoted by $(A+B)^{-1} 0$. If $H=R^{m}$, then the problem (1.1) becomes the generalized equation introduced by Robinson [1]. If $A=0$, then the problem (1.1) becomes the inclusion problem introduced by Rockafellar [2]. It is well known that the problem (1.1) is among the most interesting and intensively studied classes of mathematical problems and has wide applications in the fields of optimization and control, economics and transportation equilibrium, engineering science, and many others. For the past years, many existence results and iterative algorithms for various variational inequality and variational inclusion problems have been extended and generalized in various directions using novel and innovative techniques. A useful and important generalization is called the general variational inclusion involving the sum of two nonlinear operators. Moudafi and Noor [3] studied the sensitivity analysis of variational inclusions by using the technique of resolvent equations. Recently much attention has been given to developing iterative algorithms for solving the variational inclusions. Dong et al. [4] analyzed the solution's sensitivity for variational inequalities and variational inclusions by using a 
resolvent operator technique. By using the concept and technique of resolvent operators, Agarwal et al. [5] and Jeong [6] introduced and studied a new system of parametric generalized nonlinear mixed quasi-variational inclusions in a Hilbert space. Lan [7] introduced and studied a stable iteration procedure for a class of generalized mixed quasi-variational inclusion systems in Hilbert spaces. Recently, Zhang et al. [8] introduced a new iterative scheme for finding a common element of the set of solutions to the problem (1.1) and the set of fixed points of nonexpansive mappings in Hilbert spaces. Peng et al. [9] introduced another iterative scheme by the viscosity approximate method for finding a common element of the set of solutions of a variational inclusion with set-valued maximal monotone mapping and inverse strongly monotone mappings, the set of solutions of an equilibrium problem, and the set of fixed points of a nonexpansive mapping. For some related work, see [9-23] and the references therein.

Recently, Takahashi et al. [24] introduced the following iterative algorithm for finding a zero of the sum of two monotone operators and a fixed point of a nonexpansive mapping:

$$
x_{n+1}=\beta_{n} x_{n}+\left(1-\beta_{n}\right) S\left(\alpha_{n} x+\left(1-\alpha_{n}\right) J_{\lambda_{n}}^{B}\left(x_{n}-\lambda_{n} A x_{n}\right)\right)
$$

for all $n \geq 0$. Under some assumptions, they proved that the sequence $\left\{x_{n}\right\}$ converges strongly to a point of $F(S) \cap(A+B)^{-1} 0$.

Remark 1.1 We note that the algorithm (1.2) cannot be used to find the minimum-norm element due to the facts that $x \in C$ and $S$ is a self-mapping of $C$. However, there exist a large number of problems for which one needs to find the minimum-norm solution (see, for example, [25-29]). A useful path to circumvent this problem is to use projection. Bauschke and Browein [30] and Censor and Zenios [31] provide reviews of the field. The main difficulty is in the computation. Hence it is an interesting problem to find the minimum-norm element without using the projection.

Motivated and inspired by the works in this field, we first suggest the following two algorithms without using projection:

$$
x_{t}=(1-\kappa) S x_{t}+\kappa J_{\lambda}^{B}\left(t \gamma f\left(x_{t}\right)+(1-t) x_{t}-\lambda A x_{t}\right)
$$

for all $t \in(0,1)$ and

$$
x_{n+1}=(1-\kappa) S x_{n}+\kappa J_{\lambda_{n}}^{B}\left(\alpha_{n} \gamma f\left(x_{n}\right)+\left(1-\alpha_{n}\right) x_{n}-\lambda_{n} A x_{n}\right)
$$

for all $n \geq 0$. Notice that these two algorithms are indeed well defined (see the next section). We show that the suggested algorithms converge strongly to a point $\tilde{x}=$ $P_{F(S) \cap(A+B)^{-1} 0}(\gamma f(\tilde{x}))$ which solves the following variational inequality:

$$
\langle\gamma f(\tilde{x})-\tilde{x}, \tilde{x}-z\rangle \geq 0
$$

for all $z \in F(S) \cap(A+B)^{-1} 0$. 
As special cases, we can approach the minimum-norm element in $F(S) \cap(A+B)^{-1} 0$ without using the metric projection and give some applications.

\section{Preliminaries}

Let $H$ be a real Hilbert space with the inner product $\langle\cdot, \cdot\rangle$ and the norm $\|\cdot\|$, respectively. Let $C$ be a nonempty closed convex subset of $H$.

(1) A mapping $S: C \rightarrow C$ is said to be nonexpansive if

$$
\|S x-S y\| \leq\|x-y\|
$$

for all $x, y \in C$. We denote by $F(S)$ the set of fixed points of $S$.

(2) A mapping $A: C \rightarrow H$ is said to be $\alpha$-inverse strongly monotone if there exists $\alpha>0$ such that

$$
\langle A x-A y, x-y\rangle \geq \alpha\|A x-A y\|^{2}
$$

for all $x, y \in C$.

It is well known that, if $\mathrm{A}$ is $\alpha$-inverse strongly monotone, then $\|A x-A y\| \leq \frac{1}{\alpha}\|x-y\|$ for all $x, y \in C$.

Let $B$ be a mapping from $H$ into $2^{H}$. The effective domain of $B$ is denoted by $\operatorname{dom}(B)$, that is, $\operatorname{dom}(B)=\{x \in H: B x \neq \emptyset\}$.

(3) A multi-valued mapping $B$ is said to be a monotone operator on $H$ if

$$
\langle x-y, u-v\rangle \geq 0
$$

for all $x, y \in \operatorname{dom}(B), u \in B x$, and $v \in B y$.

(4) A monotone operator $B$ on $H$ is said to be maximal if its graph is not strictly contained in the graph of any other monotone operator on $H$.

Let $B$ be a maximal monotone operator on $H$ and $B^{-1} 0=\{x \in H: 0 \in B x\}$. For a maximal monotone operator $B$ on $H$ and $\lambda>0$, we may define a single-valued operator $J_{\lambda}^{B}=(I+$ $\lambda B)^{-1}: H \rightarrow \operatorname{dom}(B)$, which is called the resolvent of $B$ for $\lambda$. It is well known that the resolvent $J_{\lambda}^{B}$ is firmly nonexpansive, i.e.,

$$
\left\|J_{\lambda}^{B} x-J_{\lambda}^{B} y\right\|^{2} \leq\left\langle J_{\lambda}^{B} x-J_{\lambda}^{B} y, x-y\right\rangle
$$

for all $x, y \in C$ and $B^{-1} 0=F\left(J_{\lambda}^{B}\right)$ for all $\lambda>0$. The following resolvent identity is well known: for any $\lambda>0$ and $\mu>0$, the following identity holds:

$$
J_{\lambda}^{B} x=J_{\mu}^{B}\left(\frac{\mu}{\lambda} x+\left(1-\frac{\mu}{\lambda}\right) J_{\lambda}^{B} x\right)
$$

for all $x \in H$.

We use the notation that $x_{n} \rightarrow x$ stands for the weak convergence of $\left(x_{n}\right)$ to $x$ and $x_{n} \rightarrow x$ stands for the strong convergence of $\left(x_{n}\right)$ to $x$, respectively.

We need the following lemmas for the next section. 
Lemma 2.1 ([32]) Let C be a nonempty closed convex subset of a real Hilbert space H. Let $A: C \rightarrow H$ be an $\alpha$-inverse strongly monotone mapping and $\lambda>0$ be a constant. Then we have

$$
\|(I-\lambda A) x-(I-\lambda A) y\|^{2} \leq\|x-y\|^{2}+\lambda(\lambda-2 \alpha)\|A x-A y\|^{2}
$$

for all $x, y \in C$. In particular, if $0 \leq \lambda \leq 2 \alpha$, then $I-\lambda A$ is nonexpansive.

Lemma 2.2 ([33]) Let $C$ be a closed convex subset of a Hilbert space H. Let $S: C \rightarrow C$ be a nonexpansive mapping. Then $F(S)$ is a closed convex subset of $C$ and the mapping $I-S$ is demiclosed at 0 , i.e. whenever $\left\{x_{n}\right\} \subset C$ is such that $x_{n} \rightarrow x$ and $(I-S) x_{n} \rightarrow 0$, then $(I-S) x=0$.

Lemma 2.3 ([1]) Let C be a nonempty closed convex subset of a real Hilbert space H. Assume that the mapping $F: C \rightarrow H$ is monotone and weakly continuous along segments, that is, $F(x+t y) \rightarrow F(x)$ weakly as $t \rightarrow 0$. Then the variational inequality

$$
x^{*} \in C, \quad\left\langle F x^{*}, x-x^{*}\right| \geq 0
$$

for all $x \in C$ is equivalent to the dual variational inequality

$$
x^{*} \in C, \quad\left\langle F x, x-x^{*}\right\rangle \geq 0
$$

for all $x \in C$.

Lemma 2.4 ([34]) Let $\left\{x_{n}\right\},\left\{y_{n}\right\}$ be bounded sequences in a Banach space $X$ and $\left\{\beta_{n}\right\}$ be a sequence in $[0,1]$ with

$$
0<\liminf _{n \rightarrow \infty} \beta_{n} \leq \limsup _{n \rightarrow \infty} \beta_{n}<1 .
$$

Suppose that $x_{n+1}=\left(1-\beta_{n}\right) y_{n}+\beta_{n} x_{n}$ for all $n \geq 0$ and

$$
\limsup _{n \rightarrow \infty}\left(\left\|y_{n+1}-y_{n}\right\|-\left\|x_{n+1}-x_{n}\right\|\right) \leq 0 .
$$

Then $\lim _{n \rightarrow \infty}\left\|y_{n}-x_{n}\right\|=0$.

Lemma 2.5 ([35]) Assume that $\left\{a_{n}\right\}$ is a sequence of nonnegative real numbers such that

$$
a_{n+1} \leq\left(1-\gamma_{n}\right) a_{n}+\delta_{n} \gamma_{n}
$$

for all $n \geq 1$, where $\left\{\gamma_{n}\right\}$ is a sequence in $(0,1)$ and $\left\{\delta_{n}\right\}$ is a sequence such that

(a) $\sum_{n=1}^{\infty} \gamma_{n}=\infty$;

(b) $\lim \sup _{n \rightarrow \infty} \delta_{n} \leq 0$ or $\sum_{n=1}^{\infty}\left|\delta_{n} \gamma_{n}\right|<\infty$.

Then $\lim _{n \rightarrow \infty} a_{n}=0$. 


\section{Main results}

In this section, we prove our main results.

Theorem 3.1 Let $C$ be a nonempty closed convex subset of a real Hilbert space $H$. Let $A$ be an $\alpha$-inverse strongly monotone mapping from $C$ into $H$. Let $f: C \rightarrow H$ be a $\rho$-contraction and $\gamma$ be a constant such that $0<\gamma<\frac{1}{\rho}$. Let $B$ be a maximal monotone operator on $H$ such that the domain of $B$ is included in $C$. Let $J_{\lambda}^{B}=(I+\lambda B)^{-1}$ be the resolvent of $B$ for any $\lambda>0$ and $S$ be a nonexpansive mapping from $C$ into itself such that $F(S) \cap(A+B)^{-1} 0 \neq \emptyset$. Let $\lambda$ and $\kappa$ be two constants satisfying $a \leq \lambda \leq b$, where $[a, b] \subset(0,2 \alpha)$ and $\kappa \in(0,1)$. For any $t \in\left(0,1-\frac{\lambda}{2 \alpha}\right)$, let $\left\{x_{t}\right\} \subset C$ be a net generated by

$$
x_{t}=(1-\kappa) S x_{t}+\kappa J_{\lambda}^{B}\left(t \gamma f\left(x_{t}\right)+(1-t) x_{t}-\lambda A x_{t}\right) .
$$

Then the net $\left\{x_{t}\right\}$ converges strongly as $t \rightarrow 0+$ to a point $\tilde{x}=P_{F(S) \cap(A+B)^{-1} 0}(\gamma f(\tilde{x}))$, which solves the following variational inequality:

$$
\langle\gamma f(\tilde{x})-\tilde{x}, \tilde{x}-z\rangle \geq 0
$$

for all $z \in F(S) \cap(A+B)^{-1} 0$.

Proof First, we show that the net $\left\{x_{t}\right\}$ is well defined. For any $t \in\left(0,1-\frac{\lambda}{2 \alpha}\right)$, we define a mapping $W:=(1-\kappa) S+\kappa J_{\lambda}^{B}(t \gamma f+(1-t) I-\lambda A)$. Note that $J_{\lambda}^{B}, S$, and $I-\frac{\lambda}{1-t} A$ (see Lemma 2.1) are nonexpansive. For any $x, y \in C$, we have

$$
\begin{aligned}
\| W x & -W y \| \\
= & \|(1-\kappa)(S x-S y)+\kappa\left(J_{\lambda}^{B}\left(t \gamma f(x)+(1-t)\left(I-\frac{\lambda}{1-t} A\right) x\right)\right. \\
& \left.\quad-J_{\lambda}^{B}\left(t \gamma f(y)+(1-t)\left(I-\frac{\lambda}{1-t} A\right) y\right)\right) \| \\
\leq & (1-\kappa)\|S x-S y\| \\
& +\kappa\left\|t \gamma(f(x)-f(y))+(1-t)\left[\left(I-\frac{\lambda}{1-t} A\right) x-\left(I-\frac{\lambda}{1-t} A\right) y\right]\right\| \\
\leq & (1-\kappa)\|x-y\|+\kappa t \gamma\|f(x)-f(y)\| \\
& +(1-t) \kappa\left\|\left(I-\frac{\lambda}{1-t} A\right) x-\left(I-\frac{\lambda}{1-t} A\right) y\right\| \\
\leq & (1-\kappa)\|x-y\|+t \kappa \gamma \rho\|x-y\|+(1-t) \kappa\|x-y\| \\
= & {[1-(1-\gamma \rho) \kappa t]\|x-y\|, }
\end{aligned}
$$

which implies the mapping $W$ is a contraction on $C$. We use $x_{t}$ to denote the unique fixed point of $W$ in $C$. Therefore, $\left\{x_{t}\right\}$ is well defined. Set $y_{t}=J_{\lambda}^{B} u_{t}$ and $u_{t}=\gamma f\left(x_{t}\right)+(1-t) x_{t}-$ $\lambda A x_{t}$ for all $t>0$. Taking $z \in F(S) \cap(A+B)^{-1} 0$, it is obvious that $z=S z=J_{\lambda}^{B}(z-\lambda A z)$ for all $\lambda>0$ and so

$$
z=S z=J_{\lambda}^{B}(z-\lambda A z)=J_{\lambda}^{B}\left(t z+(1-t)\left(I-\frac{\lambda}{1-t} A\right) z\right)
$$


for all $t \in\left(0,1-\frac{\lambda}{2 \alpha}\right)$. From (3.1), it follows that

$$
\begin{aligned}
\left\|x_{t}-z\right\| & =\left\|(1-\kappa)\left(S x_{t}-z\right)+\kappa\left(y_{t}-z\right)\right\| \\
& \leq(1-\kappa)\left\|S x_{t}-z\right\|+\kappa\left\|y_{t}-z\right\| \\
& \leq(1-\kappa)\left\|x_{t}-z\right\|+\kappa\left\|y_{t}-z\right\| .
\end{aligned}
$$

Hence we get $\left\|x_{t}-z\right\| \leq\left\|y_{t}-z\right\|$. Since $J_{\lambda}^{B}$ is nonexpansive, we have

$$
\begin{aligned}
\| y_{t} & -z \| \\
& =\left\|J_{\lambda}^{B}\left(t \gamma f\left(x_{t}\right)+(1-t)\left(x_{t}-\frac{\lambda}{1-t} A x_{t}\right)\right)-J_{\lambda}^{B}\left(t z+(1-t)\left(z-\frac{\lambda}{1-t} A z\right)\right)\right\| \\
& \leq\left\|\left(t \gamma f\left(x_{t}\right)+(1-t)\left(x_{t}-\frac{\lambda}{1-t} A x_{t}\right)\right)-\left(t z+(1-t)\left(z-\frac{\lambda}{1-t} A z\right)\right)\right\| \\
& =\left\|(1-t)\left(\left(x_{t}-\frac{\lambda}{1-t} A x_{t}\right)-\left(z-\frac{\lambda}{1-t} A z\right)\right)+t\left(\gamma f\left(x_{t}\right)-z\right)\right\| \\
& \leq(1-t)\left\|\left(I-\frac{\lambda}{1-t} A\right) x_{t}-\left(I-\frac{\lambda}{1-t} A\right) z\right\|+t \gamma\left\|f\left(x_{t}\right)-f(z)\right\|+t\|\gamma f(z)-z\| \\
& \leq(1-t)\left\|x_{t}-z\right\|+t \gamma \rho\left\|x_{t}-z\right\|+t\|\gamma f(z)-z\| .
\end{aligned}
$$

Thus it follows that

$$
\left\|x_{t}-z\right\| \leq \frac{1}{1-\gamma \rho}\|\gamma f(z)-z\| .
$$

Therefore, $\left\{x_{t}\right\}$ is bounded. We deduce immediately that $\left\{f\left(x_{t}\right)\right\},\left\{A x_{t}\right\},\left\{S x_{t}\right\},\left\{u_{t}\right\}$, and $\left\{y_{t}\right\}$ are also bounded. By using the convexity of $\|\cdot\|$ and the $\alpha$-inverse strong monotonicity of $A$, from (3.2), we derive

$$
\begin{aligned}
\| x_{t}- & z \|^{2} \\
\leq & \left\|y_{t}-z\right\|^{2} \\
\leq & \left\|(1-t)\left(\left(x_{t}-\frac{\lambda}{1-t} A x_{t}\right)-\left(z-\frac{\lambda}{1-t} A z\right)\right)+t\left(\gamma f\left(x_{t}\right)-z\right)\right\|^{2} \\
\leq & (1-t)\left\|\left(x_{t}-\frac{\lambda}{1-t} A x_{t}\right)-\left(z-\frac{\lambda}{1-t} A z\right)\right\|^{2}+t\left\|\gamma f\left(x_{t}\right)-z\right\|^{2} \\
= & (1-t)\left\|\left(x_{t}-z\right)-\frac{\lambda}{1-t}\left(A x_{t}-A z\right)\right\|^{2}+t\left\|\gamma f\left(x_{t}\right)-z\right\|^{2} \\
= & (1-t)\left(\left\|x_{t}-z\right\|^{2}-\frac{2 \lambda}{1-t}\left\langle A x_{t}-A z, x_{t}-z\right\rangle+\frac{\lambda^{2}}{(1-t)^{2}}\left\|A x_{t}-A z\right\|^{2}\right) \\
& +t\left\|\gamma f\left(x_{t}\right)-z\right\|^{2} \\
\leq & (1-t)\left(\left\|x_{t}-z\right\|^{2}-\frac{2 \alpha \lambda}{1-t}\left\|A x_{t}-A z\right\|^{2}+\frac{\lambda^{2}}{(1-t)^{2}}\left\|A x_{t}-A z\right\|^{2}\right) \\
& +t\left\|\gamma f\left(x_{t}\right)-z\right\|^{2}
\end{aligned}
$$




$$
\begin{aligned}
& =(1-t)\left(\left\|x_{t}-z\right\|^{2}+\frac{\lambda}{(1-t)^{2}}(\lambda-2(1-t) \alpha)\left\|A x_{t}-A z\right\|^{2}\right)+t\left\|\gamma f\left(x_{t}\right)-z\right\|^{2} \\
& \leq(1-t)\left\|x_{t}-z\right\|^{2}+\frac{\lambda}{(1-t)}(\lambda-2(1-t) \alpha)\left\|A x_{t}-A z\right\|^{2}+t\left\|\gamma f\left(x_{t}\right)-z\right\|^{2}
\end{aligned}
$$

and so

$$
\frac{\lambda}{(1-t)}(2(1-t) \alpha-\lambda)\left\|A x_{t}-A z\right\|^{2} \leq t\left\|\gamma f\left(x_{t}\right)-z\right\|^{2}-t\left\|x_{t}-z\right\|^{2} \rightarrow 0 .
$$

By the assumption, we have $2(1-t) \alpha-\lambda>0$ for all $t \in\left(0,1-\frac{\lambda}{2 \alpha}\right)$ and so we obtain

$$
\lim _{t \rightarrow 0+}\left\|A x_{t}-A z\right\|=0
$$

Next, we show $\left\|x_{t}-S x_{t}\right\| \rightarrow 0$. By using the firm nonexpansivity of $J_{\lambda}^{B}$, we have

$$
\begin{aligned}
\left\|y_{t}-z\right\|^{2}= & \left\|J_{\lambda}^{B}\left(t \gamma f\left(x_{t}\right)+(1-t) x_{t}-\lambda A x_{t}\right)-z\right\|^{2} \\
= & \left\|J_{\lambda}^{B}\left(t \gamma f\left(x_{t}\right)+(1-t) x_{t}-\lambda A x_{t}\right)-J_{\lambda}^{B}(z-\lambda A z)\right\|^{2} \\
\leq & \left\langle t \gamma f\left(x_{t}\right)+(1-t) x_{t}-\lambda A x_{t}-(z-\lambda A z), y_{t}-z\right\rangle \\
= & \frac{1}{2}\left(\left\|t \gamma f\left(x_{t}\right)+(1-t) x_{t}-\lambda A x_{t}-(z-\lambda A z)\right\|^{2}+\left\|y_{t}-z\right\|^{2}\right. \\
& \left.-\left\|t \gamma f\left(x_{t}\right)+(1-t) x_{t}-\lambda\left(A x_{t}-A z\right)-y_{t}\right\|^{2}\right) .
\end{aligned}
$$

Thus it follows that

$$
\begin{aligned}
\left\|y_{t}-z\right\|^{2} \leq & \left\|t \gamma f\left(x_{t}\right)+(1-t) x_{t}-\lambda A x_{t}-(z-\lambda A z)\right\|^{2} \\
& -\left\|t \gamma f\left(x_{t}\right)+(1-t) x_{t}-\lambda\left(A x_{t}-A z\right)-y_{t}\right\|^{2} .
\end{aligned}
$$

By the nonexpansivity of $I-\frac{\lambda}{1-t} A$, we have

$$
\begin{aligned}
& \left\|t \gamma f\left(x_{t}\right)+(1-t) x_{t}-\lambda A x_{t}-(z-\lambda A z)\right\|^{2} \\
& \quad=\left\|(1-t)\left(\left(x_{t}-\frac{\lambda}{1-t} A x_{t}\right)-\left(z-\frac{\lambda}{1-t} A z\right)\right)+t\left(\gamma f\left(x_{t}\right)-z\right)\right\|^{2} \\
& \quad \leq(1-t)\left\|\left(x_{t}-\frac{\lambda}{1-t} A x_{t}\right)-\left(z-\frac{\lambda}{1-t} A z\right)\right\|^{2}+t\left\|\gamma f\left(x_{t}\right)-z\right\|^{2} \\
& \quad \leq(1-t)\left\|x_{t}-z\right\|^{2}+t\left\|\gamma f\left(x_{t}\right)-z\right\|^{2}
\end{aligned}
$$

and thus

$$
\begin{aligned}
\left\|x_{t}-z\right\|^{2} \leq & \left\|y_{t}-z\right\|^{2} \\
\leq & (1-t)\left\|x_{t}-z\right\|^{2}+t\left\|\gamma f\left(x_{t}\right)-z\right\|^{2} \\
& \quad-\left\|t \gamma f\left(x_{t}\right)+(1-t) x_{t}-\lambda\left(A x_{t}-A z\right)-y_{t}\right\|^{2} .
\end{aligned}
$$

Hence it follows that

$$
\left\|t \gamma f\left(x_{t}\right)+(1-t) x_{t}-\lambda\left(A x_{t}-A z\right)-y_{t}\right\|^{2} \leq t\left\|\gamma f\left(x_{t}\right)-z\right\|^{2} \rightarrow 0 .
$$


Since $\left\|A x_{t}-A z\right\| \rightarrow 0$, we deduce $\lim _{t \rightarrow 0+}\left\|x_{t}-y_{t}\right\|=0$, which implies that

$$
\lim _{t \rightarrow 0+}\left\|x_{t}-S x_{t}\right\|=0
$$

From (3.2), we have

$$
\begin{aligned}
\| y_{t}- & z \|^{2} \\
\leq & \left\|(1-t)\left(\left(x_{t}-\frac{\lambda}{1-t} A x_{t}\right)-\left(z-\frac{\lambda}{1-t} A z\right)\right)+t\left(\gamma f\left(x_{t}\right)-z\right)\right\|^{2} \\
= & (1-t)^{2}\left\|\left(x_{t}-\frac{\lambda}{1-t} A x_{t}\right)-\left(z-\frac{\lambda}{1-t} A z\right)\right\|^{2} \\
& +2 t(1-t)\left\langle\gamma f\left(x_{t}\right)-z,\left(x_{t}-\frac{\lambda}{1-t} A x_{t}\right)-\left(z-\frac{\lambda}{1-t} A z\right)\right\rangle+t^{2}\left\|\gamma f\left(x_{t}\right)-z\right\|^{2} \\
\leq & (1-t)^{2}\left\|x_{t}-z\right\|^{2}+2 t(1-t)\left\langle\gamma f\left(x_{t}\right)-z, x_{t}-\frac{\lambda}{1-t}\left(A x_{t}-A z\right)-z\right\rangle \\
& +t^{2}\left\|\gamma f\left(x_{t}\right)-z\right\|^{2} \\
= & (1-t)^{2}\left\|x_{t}-z\right\|^{2}+2 t(1-t) \gamma\left\langle f\left(x_{t}\right)-f(z), x_{t}-\frac{\lambda}{1-t}\left(A x_{t}-A z\right)-z\right\rangle \\
& +2 t(1-t)\left\langle\gamma f(z)-z, x_{t}-\frac{\lambda}{1-t}\left(A x_{t}-A z\right)-z\right\rangle+t^{2}\left\|\gamma f\left(x_{t}\right)-z\right\|^{2} .
\end{aligned}
$$

Note that $\left\|x_{t}-z\right\| \leq\left\|y_{t}-z\right\|$. Then we obtain

$$
\begin{aligned}
\left\|x_{t}-z\right\|^{2} \leq & (1-t)^{2}\left\|x_{t}-z\right\|^{2}+2 t(1-t) \gamma\left\|f\left(x_{t}\right)-f(z)\right\|\left(\left\|x_{t}-z\right\|+\left\|\frac{\lambda}{1-t}\left(A x_{t}-A z\right)\right\|\right) \\
& +2 t(1-t)\left\langle\gamma f(z)-z, x_{t}-\frac{\lambda}{1-t}\left(A x_{t}-A z\right)-z\right\rangle+t^{2}\left\|\gamma f\left(x_{t}\right)-z\right\|^{2} \\
\leq & (1-t)^{2}\left\|x_{t}-z\right\|^{2}+2 t(1-t) \gamma \rho\left\|x_{t}-z\right\|^{2}+2 t \lambda \gamma \rho\left\|x_{t}-z\right\|\left\|A x_{t}-A z\right\| \\
& +2 t(1-t)\left\langle\gamma f(z)-z, x_{t}-\frac{\lambda}{1-t}\left(A x_{t}-A z\right)-z\right\rangle+t^{2}\left\|\gamma f\left(x_{t}\right)-z\right\|^{2} \\
\leq & {[1-2(1-\gamma \rho) t]\left\|x_{t}-z\right\|^{2}+2 t\left[(1-t)\left\langle\gamma f(z)-z, x_{t}-\frac{\lambda}{1-t}\left(A x_{t}-A z\right)-z\right\rangle\right.} \\
& \left.+\frac{t}{2}\left(\left\|\gamma f\left(x_{t}\right)-z\right\|^{2}+\left\|x_{t}-z\right\|^{2}\right)+\lambda \gamma \rho\left\|x_{t}-z\right\|\left\|A x_{t}-A z\right\|\right] .
\end{aligned}
$$

Thus it follows that

$$
\begin{aligned}
\left\|x_{t}-z\right\|^{2} \leq & \frac{1}{1-\gamma \rho}\left(\left\langle\gamma f(z)-z, x_{t}-\frac{\lambda}{1-t}\left(A x_{t}-A z\right)-z\right\rangle\right. \\
& +\frac{t}{2}\left(\left\|\gamma f\left(x_{t}\right)-z\right\|^{2}+\left\|x_{t}-z\right\|^{2}\right)+t\|\gamma f(z)-z\|\left\|x_{t}-\frac{\lambda}{1-t}\left(A x_{t}-A z\right)-z\right\| \\
& \left.+\lambda \gamma \rho\left\|x_{t}-z\right\|\left\|A x_{t}-A z\right\|\right) \\
\leq & \frac{1}{1-\gamma \rho}\left\langle\gamma f(z)-z, x_{t}-z\right\rangle+\left(t+\left\|A x_{t}-A z\right\|\right) M
\end{aligned}
$$


where $M$ is some constant such that

$$
\begin{aligned}
& \sup \frac{1}{1-\gamma \rho}\left\{\frac{1}{2}\left(\left\|\gamma f\left(x_{t}\right)-z\right\|^{2}+\left\|x_{t}-z\right\|^{2}\right)+\|\gamma f(z)-z\|\left\|x_{t}-\frac{\lambda}{1-t}\left(A x_{t}-A z\right)-z\right\|,\right. \\
&\left.\lambda \gamma \rho\left\|x_{t}-z\right\|: t \in\left(0,1-\frac{\lambda}{2 \alpha}\right)\right\} \leq M .
\end{aligned}
$$

Next, we show that $\left\{x_{t}\right\}$ is relatively norm-compact as $t \rightarrow 0+$. Assume that $\left\{t_{n}\right\} \subset(0,1-$ $\frac{\lambda}{2 \alpha}$ ) is such that $t_{n} \rightarrow 0+$ as $n \rightarrow \infty$. Put $x_{n}:=x_{t_{n}}$. From (3.6), we have

$$
\left\|x_{n}-z\right\|^{2} \leq \frac{1}{1-\gamma \rho}\left\langle\gamma f(z)-z, x_{n}-z\right\rangle+\left(t_{n}+\left\|A x_{n}-A z\right\|\right) M .
$$

Since $\left\{x_{n}\right\}$ is bounded, without loss of generality, we may assume that $x_{n_{j}} \rightarrow \tilde{x} \in C$. Hence $y_{n_{j}}-\tilde{x}$ because of $\left\|x_{n}-y_{n}\right\| \rightarrow 0$. From (3.5), we have

$$
\lim _{n \rightarrow \infty}\left\|x_{n}-S x_{n}\right\|=0
$$

We can use Lemma 2.2 to (3.8) to deduce $\tilde{x} \in F(S)$. Further, we show that $\tilde{x}$ is also in $(A+B)^{-1} 0$. Let $v \in B u$. Note that $y_{n}=J_{\lambda}^{B}\left(t_{n} \gamma f\left(x_{n}\right)+\left(1-t_{n}\right) x_{n}-\lambda A x_{n}\right)$ for all $n \geq 1$. Then we have

$$
\begin{aligned}
& t_{n} \gamma f\left(x_{n}\right)+\left(1-t_{n}\right) x_{n}-\lambda A x_{n} \in(I+\lambda B) y_{n} \\
& \quad \Longrightarrow \quad \frac{t_{n} \gamma f\left(x_{n}\right)}{\lambda}+\frac{1-t_{n}}{\lambda} x_{n}-A x_{n}-\frac{y_{n}}{\lambda} \in B y_{n} .
\end{aligned}
$$

Since $B$ is monotone, we have, for all $(u, v) \in B$,

$$
\begin{aligned}
&\left\langle\frac{t_{n} \gamma f\left(x_{n}\right)}{\lambda}+\frac{1-t_{n}}{\lambda} x_{n}-A x_{n}-\frac{y_{n}}{\lambda}-v, y_{n}-u\right\rangle \geq 0 \\
& \Longrightarrow \quad\left\langle t_{n} \gamma f\left(x_{n}\right)+\left(1-t_{n}\right) x_{n}-\lambda A x_{n}-y_{n}-\lambda v, y_{n}-u\right\rangle \geq 0 \\
& \Longrightarrow \quad\left\langle A x_{n}+v, y_{n}-u\right\rangle \leq \frac{1}{\lambda}\left\langle x_{n}-y_{n}, y_{n}-u\right\rangle-\frac{t_{n}}{\lambda}\left\langle x_{n}-\gamma f\left(x_{n}\right), y_{n}-u\right\rangle \\
& \Longrightarrow \quad\left\langle A \tilde{x}+v, y_{n}-u\right\rangle \leq \frac{1}{\lambda}\left\langle x_{n}-y_{n}, y_{n}-u\right\rangle-\frac{t_{n}}{\lambda}\left\langle x_{n}-\gamma f\left(x_{n}\right), y_{n}-u\right\rangle \\
&+\left\langle A \tilde{x}-A x_{n}, y_{n}-u\right\rangle \\
& \Longrightarrow \quad\left\langle A \tilde{x}+v, y_{n}-u\right\rangle \leq \frac{1}{\lambda}\left\|x_{n}-y_{n}\right\|\left\|y_{n}-u\right\|+\frac{t_{n}}{\lambda}\left\|x_{n}-\gamma f\left(x_{n}\right)\right\|\left\|y_{n}-u\right\| \\
&+\left\|A \tilde{x}-A x_{n}\right\|\left\|y_{n}-u\right\| .
\end{aligned}
$$

Thus it follows that

$$
\begin{aligned}
\langle A \tilde{x}+v, \tilde{x}-u\rangle \leq & \frac{1}{\lambda}\left\|x_{n_{j}}-y_{n_{j}}\right\|\left\|y_{n_{j}}-u\right\|+\frac{t_{n_{j}}}{\lambda}\left\|x_{n_{j}}-\gamma f\left(x_{n_{j}}\right)\right\|\left\|y_{n_{j}}-u\right\| \\
& +\left\|A \tilde{x}-A x_{n_{j}}\right\|\left\|y_{n_{j}}-u\right\|+\left\langle A \tilde{x}+v, \tilde{x}-y_{n_{j}}\right\rangle .
\end{aligned}
$$

Since $\left\langle x_{n_{j}}-\tilde{x}, A x_{n_{j}}-A \tilde{x}\right\rangle \geq \alpha\left\|A x_{n_{j}}-A \tilde{x}\right\|^{2}, A x_{n_{j}} \rightarrow A z$, and $x_{n_{j}}-\tilde{x}$, it follows that $A x_{n_{j}} \rightarrow$ $A \tilde{x}$. We also observe that $t_{n} \rightarrow 0$ and $\left\|y_{n}-x_{n}\right\| \rightarrow 0$. Then, from (3.9), we can derive $\langle A \tilde{x}+$ 
$v, \tilde{x}-u\rangle \leq 0$, that is, $\langle-A \tilde{x}-v, \tilde{x}-u\rangle \geq 0$. Since $B$ is maximal monotone, we have $-A \tilde{x} \in B \tilde{x}$. This shows that $0 \in(A+B) \tilde{x}$. Hence we have $\tilde{x} \in F(S) \cap(A+B)^{-1} 0$. Therefore, substituting $\tilde{x}$ for $z$ in (3.7), we get

$$
\left\|x_{n}-\tilde{x}\right\|^{2} \leq \frac{1}{1-\gamma \rho}\left\langle\gamma f(\tilde{x})-\tilde{x}, x_{n}-\tilde{x}\right\rangle+\left(t_{n}+\left\|A x_{n}-A \tilde{x}\right\|\right) M .
$$

Consequently, the weak convergence of $\left\{x_{n}\right\}$ to $\tilde{x}$ actually implies that $x_{n} \rightarrow \tilde{x}$. This proved the relative norm-compactness of the net $\left\{x_{t}\right\}$ as $t \rightarrow 0+$.

Now, we return to (3.7) and, taking the limit as $n \rightarrow \infty$, we have

$$
\|\tilde{x}-z\|^{2} \leq \frac{1}{1-\gamma \rho}\langle\gamma f(z)-z, \tilde{x}-z\rangle
$$

for all $z \in F(S) \cap(A+B)^{-1} 0$. In particular, $\tilde{x}$ solves the following variational inequality:

$$
\tilde{x} \in F(S) \cap(A+B)^{-1} 0, \quad\langle\gamma f(z)-z, \tilde{x}-z\rangle \geq 0
$$

for all $z \in F(S) \cap(A+B)^{-1} 0$ or the equivalent dual variational inequality (see Lemma 2.3):

$$
\tilde{x} \in F(S) \cap(A+B)^{-1} 0, \quad\langle\gamma f(\tilde{x})-\tilde{x}, \tilde{x}-z\rangle \geq 0
$$

for all $z \in F(S) \cap(A+B)^{-1} 0$. Hence $\tilde{x}=P_{F(S) \cap(A+B)^{-1} 0}(\gamma f(\tilde{x}))$. Clearly, this is sufficient to conclude that the entire net $\left\{x_{t}\right\}$ converges to $\tilde{x}$. This completes the proof.

Theorem 3.2 Let $C$ be a nonempty closed convex subset of a real Hilbert space $H$. Let $A$ be an $\alpha$-inverse strongly monotone mapping from $C$ into $H$. Let $f: C \rightarrow H$ be a $\rho$-contraction and $\gamma$ be a constant such that $0<\gamma<\frac{1}{\rho}$. Let $B$ be a maximal monotone operator on $H$ such that the domain of $B$ is included in $C$. Let $J_{\lambda}^{B}=(I+\lambda B)^{-1}$ be the resolvent of $B$ for any $\lambda>0$ and $S$ be a nonexpansive mapping from $C$ into itself such that $F(S) \cap(A+B)^{-1} 0 \neq \emptyset$. For any $x_{0} \in C$, let $\left\{x_{n}\right\} \subset C$ be a sequence generated by

$$
x_{n+1}=(1-\kappa) S x_{n}+\kappa J_{\lambda_{n}}^{B}\left(\alpha_{n} \gamma f\left(x_{n}\right)+\left(1-\alpha_{n}\right) x_{n}-\lambda_{n} A x_{n}\right)
$$

for all $n \geq 0$, where $\kappa \in(0,1),\left\{\lambda_{n}\right\} \subset(0,2 \alpha)$ and $\left\{\alpha_{n}\right\} \subset(0,1)$ satisfy the following conditions:

(a) $\lim _{n \rightarrow \infty} \alpha_{n}=0, \lim _{n \rightarrow \infty} \frac{\alpha_{n+1}}{\alpha_{n}}=1$ and $\sum_{n} \alpha_{n}=\infty$;

(b) $a\left(1-\alpha_{n}\right) \leq \lambda_{n} \leq b\left(1-\alpha_{n}\right)$, where $[a, b] \subset(0,2 \alpha)$ and $\lim _{n \rightarrow \infty} \frac{\lambda_{n+1}-\lambda_{n}}{\alpha_{n+1}}=0$.

Then the sequence $\left\{x_{n}\right\}$ converges strongly to a point $\tilde{x}=P_{F(S) \cap(A+B)^{-1} 0}(\gamma f(\tilde{x}))$, which solves the following variational inequality:

$$
\langle\gamma f(\tilde{x})-\tilde{x}, \tilde{x}-z\rangle \geq 0
$$

for all $z \in F(S) \cap(A+B)^{-1} 0$.

Proof Set $y_{n}=J_{\lambda_{n}}^{B} u_{n}, u_{n}=\alpha_{n} \gamma f\left(x_{n}\right)+\left(1-\alpha_{n}\right) x_{n}-\lambda_{n} A x_{n}$ for all $n \geq 0$. Pick up $z \in F(S) \cap$ $(A+B)^{-1} 0$. It is obvious that

$$
z=S z=J_{\lambda_{n}}^{B}\left(z-\lambda_{n} A z\right)=J_{\lambda_{n}}^{B}\left(\alpha_{n} z+\left(1-\alpha_{n}\right)\left(z-\frac{\lambda_{n}}{1-\alpha_{n}} A z\right)\right)
$$


for all $n \geq 0$. Since $J_{\lambda_{n}}^{B}, S$, and $I-\frac{\lambda_{n}}{1-\alpha_{n}} A$ are nonexpansive for all $\lambda>0$ and $n \geq 1$, we have

$$
\begin{aligned}
\| y_{n}- & z \| \\
= & \left\|J_{\lambda_{n}}^{B}\left(\alpha_{n} \gamma f\left(x_{n}\right)+\left(1-\alpha_{n}\right) x_{n}-\lambda_{n} A x_{n}\right)-z\right\| \\
= & \| J_{\lambda_{n}}^{B}\left(\alpha_{n} \gamma f\left(x_{n}\right)+\left(1-\alpha_{n}\right)\left(x_{n}-\frac{\lambda_{n}}{1-\alpha_{n}} A x_{n}\right)\right) \\
& -J_{\lambda_{n}}^{B}\left(\alpha_{n} z+\left(1-\alpha_{n}\right)\left(z-\frac{\lambda_{n}}{1-\alpha_{n}} A z\right)\right) \| \\
\leq & \|\left(\alpha_{n} \gamma f\left(x_{n}\right)+\left(1-\alpha_{n}\right)\left(x_{n}-\frac{\lambda_{n}}{1-\alpha_{n}} A x_{n}\right)\right) \\
& -\left(\alpha_{n} z+\left(1-\alpha_{n}\right)\left(z-\frac{\lambda_{n}}{1-\alpha_{n}} A z\right)\right) \|^{2} \\
= & \left\|\left(1-\alpha_{n}\right)\left(\left(x_{n}-\frac{\lambda_{n}}{1-\alpha_{n}} A x_{n}\right)-\left(z-\frac{\lambda_{n}}{1-\alpha_{n}} A z\right)\right)+\alpha_{n}\left(\gamma f\left(x_{n}\right)-z\right)\right\| \\
\leq & \left(1-\alpha_{n}\right)\left\|x_{n}-z\right\|+\alpha_{n}\left\|\gamma f\left(x_{n}\right)-\gamma f(z)\right\|+\alpha_{n}\|\gamma f(z)-z\| \\
\leq & {\left[1-(1-\gamma \rho) \alpha_{n}\right]\left\|x_{n}-z\right\|+\alpha_{n}\|\gamma f(z)-z\| . }
\end{aligned}
$$

Hence we have

$$
\begin{aligned}
\left\|x_{n+1}-z\right\| & \leq(1-\kappa)\left\|S x_{n}-z\right\|+\kappa\left\|y_{n}-z\right\| \\
& \leq(1-\kappa)\left\|x_{n}-z\right\|+\kappa\left[1-(1-\gamma \rho) \alpha_{n}\right]\left\|x_{n}-z\right\|+\kappa \alpha_{n}\|\gamma f(z)-z\| \\
& =\left[1-(1-\gamma \rho) \kappa \alpha_{n}\right]\left\|x_{n}-z\right\|+\kappa \alpha_{n}\|\gamma f(z)-z\| .
\end{aligned}
$$

By induction, we have

$$
\left\|x_{n+1}-z\right\| \leq \max \left\{\left\|x_{0}-z\right\|, \frac{1}{1-\gamma \rho}\|\gamma f(z)-z\|\right\}
$$

Therefore, $\left\{x_{n}\right\}$ is bounded. Since $A$ is $\alpha$-inverse strongly monotone, it is $\frac{1}{\alpha}$-Lipschitz continuous. We deduce immediately that $\left\{f\left(x_{n}\right)\right\},\left\{S x_{n}\right\},\left\{A x_{n}\right\},\left\{u_{n}\right\}$, and $\left\{y_{n}\right\}$ are also bounded. By using the convexity of $\|\cdot\|$ and the $\alpha$-inverse strong monotonicity of $A$, it follows from (3.11) that

$$
\begin{aligned}
\left\|\left(1-\alpha_{n}\right)\left(\left(x_{n}-\frac{\lambda_{n}}{1-\alpha_{n}} A x_{n}\right)-\left(z-\frac{\lambda_{n}}{1-\alpha_{n}} A z\right)\right)+\alpha_{n}\left(\gamma f\left(x_{n}\right)-z\right)\right\|^{2} \\
\leq\left(1-\alpha_{n}\right)\left\|\left(x_{n}-\frac{\lambda_{n}}{1-\alpha_{n}} A x_{n}\right)-\left(z-\frac{\lambda_{n}}{1-\alpha_{n}} A z\right)\right\|^{2}+\alpha_{n}\left\|\gamma f\left(x_{n}\right)-z\right\|^{2} \\
=\left(1-\alpha_{n}\right)\left\|\left(x_{n}-z\right)-\frac{\lambda_{n}}{1-\alpha_{n}}\left(A x_{n}-A z\right)\right\|^{2}+\alpha_{n}\left\|\gamma f\left(x_{n}\right)-z\right\|^{2} \\
=\left(1-\alpha_{n}\right)\left(\left\|x_{n}-z\right\|^{2}-\frac{2 \lambda_{n}}{1-\alpha_{n}}\left\langle A x_{n}-A z, x_{n}-z\right\rangle+\frac{\lambda_{n}^{2}}{\left(1-\alpha_{n}\right)^{2}}\left\|A x_{n}-A z\right\|^{2}\right) \\
\quad+\alpha_{n}\left\|\gamma f\left(x_{n}\right)-z\right\|^{2}
\end{aligned}
$$




$$
\begin{aligned}
\leq & \left(1-\alpha_{n}\right)\left(\left\|x_{n}-z\right\|^{2}-\frac{2 \alpha \lambda_{n}}{1-\alpha_{n}}\left\|A x_{n}-A z\right\|^{2}+\frac{\lambda_{n}^{2}}{\left(1-\alpha_{n}\right)^{2}}\left\|A x_{n}-A z\right\|^{2}\right) \\
& +\alpha_{n}\left\|\gamma f\left(x_{n}\right)-z\right\|^{2} \\
= & \left(1-\alpha_{n}\right)\left(\left\|x_{n}-z\right\|^{2}+\frac{\lambda_{n}}{\left(1-\alpha_{n}\right)^{2}}\left(\lambda_{n}-2\left(1-\alpha_{n}\right) \alpha\right)\left\|A x_{n}-A z\right\|^{2}\right) \\
& +\alpha_{n}\left\|\gamma f\left(x_{n}\right)-z\right\|^{2} .
\end{aligned}
$$

By the condition (c), we get $\lambda_{n}-2\left(1-\alpha_{n}\right) \alpha \leq 0$ for all $n \geq 0$. Then, from (3.11) and (3.12), we obtain

$$
\begin{aligned}
\left\|J_{\lambda_{n}}^{B} u_{n}-z\right\|^{2} \leq & \left(1-\alpha_{n}\right)\left(\left\|x_{n}-z\right\|^{2}+\frac{\lambda_{n}}{\left(1-\alpha_{n}\right)^{2}}\left(\lambda_{n}-2\left(1-\alpha_{n}\right) \alpha\right)\left\|A x_{n}-A z\right\|^{2}\right) \\
& +\alpha_{n}\left\|\gamma f\left(x_{n}\right)-z\right\|^{2} .
\end{aligned}
$$

From (3.10), it follows that

$$
\begin{aligned}
\left\|x_{n+1}-z\right\|^{2} & =\left\|(1-\kappa)\left(S x_{n}-z\right)+\kappa\left(J_{\lambda_{n}}^{B} u_{n}-z\right)\right\|^{2} \\
& \leq(1-\kappa)\left\|x_{n}-z\right\|^{2}+\kappa\left\|J_{\lambda_{n}}^{B} u_{n}-z\right\|^{2} .
\end{aligned}
$$

Next, we estimate $\left\|x_{n+1}-x_{n}\right\|$. In fact, we have

$$
\begin{aligned}
\left\|x_{n+2}-x_{n+1}\right\| & =\left\|(1-\kappa)\left(S x_{n+1}-S x_{n}\right)+\kappa\left(y_{n+1}-y_{n}\right)\right\| \\
& \leq(1-\kappa)\left\|x_{n+1}-x_{n}\right\|+\kappa\left\|y_{n+1}-y_{n}\right\|
\end{aligned}
$$

and

$$
\begin{aligned}
\left\|y_{n+1}-y_{n}\right\|= & \left\|J_{\lambda_{n+1}}^{B} u_{n+1}-J_{\lambda_{n}}^{B} u_{n}\right\| \\
\leq & \left\|J_{\lambda_{n+1}}^{B} u_{n+1}-J_{\lambda_{n+1}}^{B} u_{n}\right\|+\left\|J_{\lambda_{n+1}}^{B} u_{n}-J_{\lambda_{n}}^{B} u_{n}\right\| \\
\leq & \|\left(\alpha_{n+1} \gamma f\left(x_{n+1}\right)+\left(1-\alpha_{n+1}\right) x_{n+1}-\lambda_{n+1} A x_{n+1}\right) \\
& -\left(\alpha_{n} \gamma f\left(x_{n}\right)+\left(1-\alpha_{n}\right) x_{n}-\lambda_{n} A x_{n}\right)\|+\| J_{\lambda_{n+1}}^{B} u_{n}-J_{\lambda_{n}}^{B} u_{n} \| \\
= & \| \alpha_{n+1} \gamma\left(f\left(x_{n+1}\right)-f\left(x_{n}\right)\right)+\left(\alpha_{n+1}-\alpha_{n}\right) \gamma f\left(x_{n}\right) \\
& +\left(1-\alpha_{n+1}\right)\left[\left(I-\frac{\lambda_{n+1}}{1-\alpha_{n+1}} A\right) x_{n+1}-\left(I-\frac{\lambda_{n+1}}{1-\alpha_{n+1}} A\right) x_{n}\right] \\
& +\left(\alpha_{n}-\alpha_{n+1}\right) x_{n}+\left(\lambda_{n}-\lambda_{n+1}\right) A x_{n}\|+\| J_{\lambda_{n+1}}^{B} u_{n}-J_{\lambda_{n}}^{B} u_{n} \| \\
\leq & \alpha_{n+1} \gamma \rho\left\|x_{n+1}-x_{n}\right\|+\left|\alpha_{n+1}-\alpha_{n}\right|\left(\left\|\gamma f\left(x_{n}\right)\right\|+\left\|x_{n}\right\|\right) \\
& +\left(1-\alpha_{n+1}\right)\left\|x_{n+1}-x_{n}\right\|+\left|\lambda_{n}-\lambda_{n+1}\right|\left\|A x_{n}\right\|+\left\|J_{\lambda_{n+1}}^{B} u_{n}-J_{\lambda_{n}}^{B} u_{n}\right\| \\
= & {\left[1-(1-\gamma \rho) \alpha_{n+1}\right]\left\|x_{n+1}-x_{n}\right\|+\left|\alpha_{n+1}-\alpha_{n}\right|\left(\left\|\gamma f\left(x_{n}\right)\right\|+\left\|x_{n}\right\|\right) } \\
& +\left|\lambda_{n}-\lambda_{n+1}\right|\left\|A x_{n}\right\|+\left\|J_{\lambda_{n+1}}^{B} u_{n}-J_{\lambda_{n}}^{B} u_{n}\right\| .
\end{aligned}
$$


By the resolvent identity (2.1), we have

$$
J_{\lambda_{n+1}}^{B} u_{n}=J_{\lambda_{n}}^{B}\left(\frac{\lambda_{n}}{\lambda_{n+1}} u_{n}+\left(1-\frac{\lambda_{n}}{\lambda_{n+1}}\right) J_{\lambda_{n+1}}^{B} u_{n}\right) .
$$

Thus it follows that

$$
\begin{aligned}
\left\|J_{\lambda_{n+1}}^{B} u_{n}-J_{\lambda_{n}}^{B} u_{n}\right\| & =\left\|J_{\lambda_{n}}^{B}\left(\frac{\lambda_{n}}{\lambda_{n+1}} u_{n}+\left(1-\frac{\lambda_{n}}{\lambda_{n+1}}\right) J_{\lambda_{n+1}}^{B} u_{n}\right)-J_{\lambda_{n}}^{B} u_{n}\right\| \\
& \leq\left\|\left(\frac{\lambda_{n}}{\lambda_{n+1}} u_{n}+\left(1-\frac{\lambda_{n}}{\lambda_{n+1}}\right) J_{\lambda_{n+1}}^{B} u_{n}\right)-u_{n}\right\| \\
& \leq \frac{\left|\lambda_{n+1}-\lambda_{n}\right|}{\lambda_{n+1}}\left\|u_{n}-J_{\lambda_{n+1}}^{B} u_{n}\right\|
\end{aligned}
$$

and so

$$
\begin{aligned}
&\left\|x_{n+2}-x_{n+1}\right\| \\
& \leq(1-\kappa)\left\|x_{n+1}-x_{n}\right\|+\kappa\left\|y_{n+1}-y_{n}\right\| \\
& \leq(1-\kappa)\left\|x_{n+1}-x_{n}\right\|+\kappa\left[1-(1-\gamma \rho) \alpha_{n+1}\right]\left\|x_{n+1}-x_{n}\right\| \\
&+\kappa\left|\alpha_{n+1}-\alpha_{n}\right|\left(\left\|\gamma f\left(x_{n}\right)\right\|+\left\|x_{n}\right\|\right)+\kappa\left|\lambda_{n}-\lambda_{n+1}\right|\left\|A x_{n}\right\| \\
&+\kappa \frac{\left|\lambda_{n+1}-\lambda_{n}\right|}{\lambda_{n+1}}\left\|u_{n}-J_{\lambda_{n+1}}^{B} u_{n}\right\| \\
& \leq {\left[1-(1-\gamma \rho) \kappa \alpha_{n+1}\right]\left\|x_{n+1}-x_{n}\right\|+(1-\gamma \rho) \kappa \alpha_{n+1}\left[\frac{\left|\alpha_{n+1}-\alpha_{n}\right|}{\alpha_{n+1}} \frac{\left\|\gamma f\left(x_{n}\right)\right\|+\left\|x_{n}\right\|}{1-\gamma \rho}\right.} \\
&\left.+\frac{\left|\lambda_{n}-\lambda_{n+1}\right|}{\alpha_{n+1}} \frac{\left\|A x_{n}\right\|}{1-\gamma \rho}+\frac{\left|\lambda_{n+1}-\lambda_{n}\right|}{\alpha_{n+1} \lambda_{n+1}} \frac{\left\|u_{n}-J_{\lambda_{n+1}}^{B} u_{n}\right\|}{1-\gamma \rho}\right] .
\end{aligned}
$$

By the assumptions, we know that $\frac{\left|\alpha_{n+1}-\alpha_{n}\right|}{\alpha_{n+1}} \rightarrow 0$ and $\frac{\left|\lambda_{n+1}-\lambda_{n}\right|}{\alpha_{n+1}} \rightarrow 0$. Then, from Lemma 2.5, we get

$$
\lim _{n \rightarrow \infty}\left\|x_{n+1}-x_{n}\right\|=0
$$

Thus, from (3.13) and (3.14), it follows that

$$
\begin{aligned}
& \left\|x_{n+1}-z\right\|^{2} \\
& \leq(1-\kappa)\left\|x_{n}-z\right\|^{2}+\kappa\left\|J_{\lambda_{n}}^{B} u_{n}-z\right\|^{2} \\
& \leq \kappa\left[\left(1-\alpha_{n}\right)\left(\left\|x_{n}-z\right\|^{2}+\frac{\lambda_{n}}{\left(1-\alpha_{n}\right)^{2}}\left(\lambda_{n}-2\left(1-\alpha_{n}\right) \alpha\right)\left\|A x_{n}-A z\right\|^{2}\right)\right. \\
& \left.\quad+\alpha_{n}\left\|\gamma f\left(x_{n}\right)-z\right\|^{2}\right]+(1-\kappa)\left\|x_{n}-z\right\|^{2} \\
& \quad\left[1-\kappa \alpha_{n}\right]\left\|x_{n}-z\right\|^{2}+\frac{\kappa \lambda_{n}}{1-\alpha_{n}}\left(\lambda_{n}-2\left(1-\alpha_{n}\right) \alpha\right)\left\|A x_{n}-A z\right\|^{2}+\kappa \alpha_{n}\left\|\gamma f\left(x_{n}\right)-z\right\|^{2} \\
& \leq \quad\left\|x_{n}-z\right\|^{2}+\frac{\kappa \lambda_{n}}{1-\alpha_{n}}\left(\lambda_{n}-2\left(1-\alpha_{n}\right) \alpha\right)\left\|A x_{n}-A z\right\|^{2}+\kappa \alpha_{n}\left\|\gamma f\left(x_{n}\right)-z\right\|^{2}
\end{aligned}
$$


and so

$$
\begin{aligned}
& \frac{\kappa \lambda_{n}}{\left(1-\alpha_{n}\right)}\left(2\left(1-\alpha_{n}\right) \alpha-\lambda_{n}\right)\left\|A x_{n}-A z\right\|^{2} \\
& \quad \leq\left\|x_{n}-z\right\|^{2}-\left\|x_{n+1}-z\right\|^{2}+\kappa \alpha_{n}\left\|\gamma f\left(x_{n}\right)-z\right\|^{2} \\
& \quad \leq\left(\left\|x_{n}-z\right\|-\left\|x_{n+1}-z\right\|\right)\left\|x_{n+1}-x_{n}\right\|+\kappa \alpha_{n}\left\|\gamma f\left(x_{n}\right)-z\right\|^{2} .
\end{aligned}
$$

Since $\lim _{n \rightarrow \infty} \alpha_{n}=0, \lim _{n \rightarrow \infty}\left\|x_{n+1}-x_{n}\right\|=0$, and $\liminf _{n \rightarrow \infty} \frac{\kappa \lambda_{n}}{\left(1-\alpha_{n}\right)}\left(2\left(1-\alpha_{n}\right) \alpha-\lambda_{n}\right)>0$, we have

$$
\lim _{n \rightarrow \infty}\left\|A x_{n}-A z\right\|=0
$$

Next, we show $\left\|x_{n}-S x_{n}\right\| \rightarrow 0$. By using the firm nonexpansivity of $J_{\lambda_{n}}^{B}$, we have

$$
\begin{aligned}
\left\|J_{\lambda_{n}}^{B} u_{n}-z\right\|^{2}= & \left\|J_{\lambda_{n}}^{B}\left(\alpha_{n} \gamma f\left(x_{n}\right)+\left(1-\alpha_{n}\right) x_{n}-\lambda_{n} A x_{n}\right)-J_{\lambda_{n}}^{B}\left(z-\lambda_{n} A z\right)\right\|^{2} \\
\leq & \left\langle\alpha_{n} \gamma f\left(x_{n}\right)+\left(1-\alpha_{n}\right) x_{n}-\lambda_{n} A x_{n}-\left(z-\lambda_{n} A z\right), J_{\lambda_{n}}^{B} u_{n}-z\right\rangle \\
= & \frac{1}{2}\left(\left\|\alpha_{n} \gamma f\left(x_{n}\right)+\left(1-\alpha_{n}\right) x_{n}-\lambda_{n} A x_{n}-\left(z-\lambda_{n} A z\right)\right\|^{2}+\left\|J_{\lambda_{n}}^{B} u_{n}-z\right\|^{2}\right. \\
& \left.-\left\|\alpha_{n} \gamma f\left(x_{n}\right)+\left(1-\alpha_{n}\right) x_{n}-\lambda_{n}\left(A x_{n}-A z\right)-J_{\lambda_{n}}^{B} u_{n}\right\|^{2}\right) .
\end{aligned}
$$

From the condition (c) and the $\alpha$-inverse strongly monotonicity of $A$, we know that $I-$ $\lambda_{n} A /\left(1-\alpha_{n}\right)$ is nonexpansive. Hence it follows that

$$
\begin{aligned}
& \left\|\alpha_{n} \gamma f\left(x_{n}\right)+\left(1-\alpha_{n}\right) x_{n}-\lambda_{n} A x_{n}-\left(z-\lambda_{n} A z\right)\right\|^{2} \\
& \quad=\left\|\left(1-\alpha_{n}\right)\left(\left(x_{n}-\frac{\lambda_{n}}{1-\alpha_{n}} A x_{n}\right)-\left(z-\frac{\lambda_{n}}{1-\alpha_{n}} A z\right)\right)+\alpha_{n}\left(\gamma f\left(x_{n}\right)-z\right)\right\|^{2} \\
& \quad \leq\left(1-\alpha_{n}\right)\left\|\left(x_{n}-\frac{\lambda_{n}}{1-\alpha_{n}} A x_{n}\right)-\left(z-\frac{\lambda_{n}}{1-\alpha_{n}} A z\right)\right\|^{2}+\alpha_{n}\left\|\gamma f\left(x_{n}\right)-z\right\|^{2} \\
& \quad \leq\left(1-\alpha_{n}\right)\left\|x_{n}-z\right\|^{2}+\alpha_{n}\left\|\gamma f\left(x_{n}\right)-z\right\|^{2}
\end{aligned}
$$

and thus

$$
\begin{aligned}
\left\|J_{\lambda_{n}}^{B} u_{n}-z\right\|^{2} \leq & \frac{1}{2}\left(\left(1-\alpha_{n}\right)\left\|x_{n}-z\right\|^{2}+\alpha_{n}\left\|\gamma f\left(x_{n}\right)-z\right\|^{2}+\left\|J_{\lambda_{n}}^{B} u_{n}-z\right\|^{2}\right. \\
& \left.-\left\|\alpha_{n} \gamma f\left(x_{n}\right)+\left(1-\alpha_{n}\right) x_{n}-J_{\lambda_{n}}^{B} u_{n}-\lambda_{n}\left(A x_{n}-A z\right)\right\|^{2}\right),
\end{aligned}
$$

that is,

$$
\begin{aligned}
\| J_{\lambda_{n}}^{B} & u_{n}-z \|^{2} \\
& \leq\left(1-\alpha_{n}\right)\left\|x_{n}-z\right\|^{2}+\alpha_{n}\left\|\gamma f\left(x_{n}\right)-z\right\|^{2} \\
& \quad-\left\|\alpha_{n} \gamma f\left(x_{n}\right)+\left(1-\alpha_{n}\right) x_{n}-J_{\lambda_{n}}^{B} u_{n}-\lambda_{n}\left(A x_{n}-A z\right)\right\|^{2} \\
& =\left(1-\alpha_{n}\right)\left\|x_{n}-z\right\|^{2}+\alpha_{n}\left\|\gamma f\left(x_{n}\right)-z\right\|^{2}-\left\|\alpha_{n} \gamma f\left(x_{n}\right)+\left(1-\alpha_{n}\right) x_{n}-J_{\lambda_{n}}^{B} u_{n}\right\|^{2}
\end{aligned}
$$




$$
\begin{aligned}
& +2 \lambda_{n}\left\langle\alpha_{n} \gamma f\left(x_{n}\right)+\left(1-\alpha_{n}\right) x_{n}-J_{\lambda_{n}}^{B} u_{n}, A x_{n}-A z\right\rangle-\lambda_{n}^{2}\left\|A x_{n}-A z\right\|^{2} \\
\leq & \left(1-\alpha_{n}\right)\left\|x_{n}-z\right\|^{2}+\alpha_{n}\left\|\gamma f\left(x_{n}\right)-z\right\|^{2}-\left\|\alpha_{n} \gamma f\left(x_{n}\right)+\left(1-\alpha_{n}\right) x_{n}-J_{\lambda_{n}}^{B} u_{n}\right\|^{2} \\
& +2 \lambda_{n}\left\|\alpha_{n} \gamma f\left(x_{n}\right)+\left(1-\alpha_{n}\right) x_{n}-J_{\lambda_{n}}^{B} u_{n}\right\|\left\|A x_{n}-A z\right\| .
\end{aligned}
$$

This together with (3.14) implies that

$$
\begin{aligned}
\left\|x_{n+1}-z\right\|^{2} \leq & (1-\kappa)\left\|x_{n}-z\right\|^{2}+\kappa\left(1-\alpha_{n}\right)\left\|x_{n}-z\right\|^{2}+\kappa \alpha_{n}\left\|\gamma f\left(x_{n}\right)-z\right\|^{2} \\
& -\kappa\left\|\alpha_{n} \gamma f\left(x_{n}\right)+\left(1-\alpha_{n}\right) x_{n}-J_{\lambda_{n}}^{B} u_{n}\right\|^{2} \\
& +2 \lambda_{n} \kappa\left\|\alpha_{n} \gamma f\left(x_{n}\right)+\left(1-\alpha_{n}\right) x_{n}-J_{\lambda_{n}}^{B} u_{n}\right\|\left\|A x_{n}-A z\right\| \\
= & {\left[1-\kappa \alpha_{n}\right]\left\|x_{n}-z\right\|^{2}+\kappa \alpha_{n}\left\|\gamma f\left(x_{n}\right)-z\right\|^{2} } \\
& -\kappa\left\|\alpha_{n} \gamma f\left(x_{n}\right)+\left(1-\alpha_{n}\right) x_{n}-J_{\lambda_{n}}^{B} u_{n}\right\|^{2} \\
& +2 \lambda_{n} \kappa\left\|\alpha_{n} \gamma f\left(x_{n}\right)+\left(1-\alpha_{n}\right) x_{n}-J_{\lambda_{n}}^{B} u_{n}\right\|\left\|A x_{n}-A z\right\|
\end{aligned}
$$

and hence

$$
\begin{aligned}
\kappa\left\|\alpha_{n} \gamma f\left(x_{n}\right)+\left(1-\alpha_{n}\right) x_{n}-J_{\lambda_{n}}^{B} u_{n}\right\|^{2} \\
\leq \quad\left\|x_{n}-z\right\|^{2}-\left\|x_{n+1}-z\right\|^{2}-\kappa \alpha_{n}\left\|x_{n}-z\right\|^{2}+\kappa \alpha_{n}\left\|\gamma f\left(x_{n}\right)-z\right\|^{2} \\
\quad+2 \lambda_{n} \kappa\left\|\alpha_{n} \gamma f\left(x_{n}\right)+\left(1-\alpha_{n}\right) x_{n}-J_{\lambda_{n}}^{B} u_{n}\right\|\left\|A x_{n}-A z\right\| \\
\leq\left(\left\|x_{n}-z\right\|+\left\|x_{n+1}-z\right\|\right)\left\|x_{n+1}-x_{n}\right\|+\kappa \alpha_{n}\left\|\gamma f\left(x_{n}\right)-z\right\|^{2} \\
\quad+2 \lambda_{n} \kappa\left\|\alpha_{n} \gamma f\left(x_{n}\right)+\left(1-\alpha_{n}\right) x_{n}-J_{\lambda_{n}}^{B} u_{n}\right\|\left\|A x_{n}-A z\right\| .
\end{aligned}
$$

Since $\left\|x_{n+1}-x_{n}\right\| \rightarrow 0, \alpha_{n} \rightarrow 0$, and $\left\|A x_{n}-A z\right\| \rightarrow 0$ (by (3.16)), we deduce

$$
\lim _{n \rightarrow \infty}\left\|\alpha_{n} \gamma f\left(x_{n}\right)+\left(1-\alpha_{n}\right) x_{n}-J_{\lambda_{n}}^{B} u_{n}\right\|=0
$$

This implies that

$$
\lim _{n \rightarrow \infty}\left\|x_{n}-J_{\lambda_{n}}^{B} u_{n}\right\|=0
$$

Combining (3.10), (3.15), and (3.17), we get

$$
\lim _{n \rightarrow \infty}\left\|x_{n}-S x_{n}\right\|=0
$$

Put $\tilde{x}=\lim _{t \rightarrow 0+} x_{t}=P_{F(S) \cap(A+B)^{-1} 0}(\gamma f(\tilde{x}))$, where $\left\{x_{t}\right\}$ is the net defined by (3.1).

Finally, we show that $x_{n} \rightarrow \tilde{x}$. Taking $z=\tilde{x}$ in (3.16), we get $\left\|A x_{n}-A \tilde{x}\right\| \rightarrow 0$. First, we prove $\lim \sup _{n \rightarrow \infty}\left\langle\gamma f(\tilde{x})-\tilde{x}, x_{n}-\tilde{x}\right\rangle \leq 0$. We take a subsequence $\left\{x_{n_{i}}\right\}$ of $\left\{x_{n}\right\}$ such that

$$
\limsup _{n \rightarrow \infty}\left\langle\gamma f(\tilde{x})-\tilde{x}, x_{n}-\tilde{x}\right\rangle=\lim _{i \rightarrow \infty}\left\langle\gamma f(\tilde{x})-\tilde{x}, x_{n_{i}}-\tilde{x}\right\rangle .
$$

There exists a subsequence $\left\{x_{n_{i_{j}}}\right\}$ of $\left\{x_{n_{i}}\right\}$ which converges weakly to a point $w \in C$. Hence $\left\{y_{n_{i j}}\right\}$ also converges weakly to $w$ because of $\left\|x_{n_{i_{j}}}-y_{n_{i_{j}}}\right\| \rightarrow 0$. By the demi-closedness 
principle of the nonexpansive mapping (see Lemma 2.2) and (3.18), we deduce $w \in F(S)$. Furthermore, by a similar argument to that of Theorem 3.1, we can show that $w$ is also in $(A+B)^{-1} 0$. Hence we have $w \in F(S) \cap(A+B)^{-1} 0$. This implies that

$$
\limsup _{n \rightarrow \infty}\left\langle\gamma f(\tilde{x})-\tilde{x}, x_{n}-\tilde{x}\right\rangle=\lim _{j \rightarrow \infty}\left\langle\gamma f(\tilde{x})-\tilde{x}, x_{n_{i_{j}}}-\tilde{x}\right\rangle=\langle\gamma f(\tilde{x})-\tilde{x}, w-\tilde{x}\rangle .
$$

Note that $\tilde{x}=P_{F(S) \cap(A+B)^{-1} 0}(\gamma f(\tilde{x}))$. Then we have

$$
\langle\gamma f(\tilde{x})-\tilde{x}, w-\tilde{x}\rangle \leq 0
$$

for all $w \in F(S) \cap(A+B)^{-1} 0$. Therefore, it follows that

$$
\limsup _{n \rightarrow \infty}\left\langle\gamma f(\tilde{x})-\tilde{x}, x_{n}-\tilde{x}\right\rangle \leq 0
$$

From (3.10), we have

$$
\begin{aligned}
&\left\|x_{n+1}-\tilde{x}\right\|^{2} \\
& \leq(1-\kappa)\left\|x_{n}-\tilde{x}\right\|^{2}+\kappa\left\|J_{\lambda_{n}}^{B} u_{n}-\tilde{x}\right\|^{2} \\
&=(1-\kappa)\left\|x_{n}-\tilde{x}\right\|^{2}+\kappa\left\|J_{\lambda_{n}}^{B} u_{n}-J_{\lambda_{n}}^{B}\left(\tilde{x}-\lambda_{n} A \tilde{x}\right)\right\|^{2} \\
& \leq(1-\kappa)\left\|x_{n}-\tilde{x}\right\|^{2}+\kappa\left\|u_{n}-\left(\tilde{x}-\lambda_{n} A \tilde{x}\right)\right\|^{2} \\
&=(1-\kappa)\left\|x_{n}-\tilde{x}\right\|^{2}+\kappa\left\|\alpha_{n} \gamma f\left(x_{n}\right)+\left(1-\alpha_{n}\right) x_{n}-\lambda_{n} A x_{n}-\left(\tilde{x}-\lambda_{n} A \tilde{x}\right)\right\|^{2} \\
&= \kappa\left\|\left(1-\alpha_{n}\right)\left(\left(x_{n}-\frac{\lambda_{n}}{1-\alpha_{n}} A x_{n}\right)-\left(\tilde{x}-\frac{\lambda_{n}}{1-\alpha_{n}} A \tilde{x}\right)\right)+\alpha_{n}\left(\gamma f\left(x_{n}\right)-\tilde{x}\right)\right\|^{2} \\
&+(1-\kappa)\left\|x_{n}-\tilde{x}\right\|^{2} \\
&=(1-\kappa)\left\|x_{n}-\tilde{x}\right\|^{2}+\kappa\left(\left(1-\alpha_{n}\right)^{2}\left\|\left(x_{n}-\frac{\lambda_{n}}{1-\alpha_{n}} A x_{n}\right)-\left(\tilde{x}-\frac{\lambda_{n}}{1-\alpha_{n}} A \tilde{x}\right)\right\|^{2}\right. \\
&+2 \alpha_{n}\left(1-\alpha_{n}\right)\left(\gamma f\left(x_{n}\right)-\tilde{x},\left(x_{n}-\frac{\lambda_{n}}{1-\alpha_{n}} A x_{n}\right)-\left(\tilde{x}-\frac{\lambda_{n}}{1-\alpha_{n}} A \tilde{x}\right)\right\rangle \\
&\left.+\alpha_{n}^{2}\left\|\gamma f\left(x_{n}\right)-\tilde{x}\right\|^{2}\right) \\
& \leq(1-\kappa)\left\|x_{n}-\tilde{x}\right\|^{2}+\kappa\left(\left(1-\alpha_{n}\right)^{2}\left\|x_{n}-\tilde{x}\right\|^{2}+2 \alpha_{n} \lambda_{n}\left(\gamma f\left(x_{n}\right)-\tilde{x}, A x_{n}-A \tilde{x}\right\rangle\right. \\
&+2 \alpha_{n}\left(1-\alpha_{n}\right) \gamma\left(f\left(x_{n}\right)-f(\tilde{x}), x_{n}-\tilde{x}\right\rangle+2 \alpha_{n}\left(1-\alpha_{n}\right)\left\langle\gamma f(\tilde{x})-\tilde{x}, x_{n}-\tilde{x}\right\rangle \\
&\left.+\alpha_{n}^{2}\left\|\gamma f\left(x_{n}\right)-\tilde{x}\right\|^{2}\right) \\
& \leq(1-\kappa)\left\|x_{n}-\tilde{x}\right\|^{2}+\kappa\left(\left(1-\alpha_{n}\right)^{2}\left\|x_{n}-\tilde{x}\right\|^{2}+2 \alpha_{n} \lambda_{n}\left\|\gamma f\left(x_{n}\right)-\tilde{x}\right\|\left\|A x_{n}-A \tilde{x}\right\|\right. \\
&\left.+2 \alpha_{n}\left(1-\alpha_{n}\right) \gamma \rho\left\|x_{n}-\tilde{x}\right\|^{2}+2 \alpha_{n}\left(1-\alpha_{n}\right)\left\langle\gamma f(\tilde{x})-\tilde{x}, x_{n}-\tilde{x}\right\rangle+\alpha_{n}^{2}\left\|\gamma f\left(x_{n}\right)-\tilde{x}\right\|^{2}\right) \\
& \leq {\left[1-2 \kappa(1-\gamma \rho) \alpha_{n}\right]\left\|x_{n}-\tilde{x}\right\|^{2}+2 \alpha_{n} \kappa \lambda_{n}\left\|\gamma f\left(x_{n}\right)-\tilde{x}\right\|\left\|A x_{n}-A \tilde{x}\right\| } \\
&+2 \alpha_{n} \kappa\left(1-\alpha_{n}\right)\left(\gamma f(\tilde{x})-\tilde{x}, x_{n}-\tilde{x}\right\rangle+\kappa \alpha_{n}^{2}\left(\left\|\gamma f\left(x_{n}\right)-\tilde{x}\right\|^{2}+\left\|x_{n}-\tilde{x}\right\|^{2}\right) \\
&= {\left[1-2 \kappa(1-\gamma \rho) \alpha_{n}\right]\left\|x_{n}-\tilde{x}\right\|^{2} } \\
& \\
&
\end{aligned}
$$




$$
\begin{aligned}
& +2 \kappa(1-\gamma \rho) \alpha_{n}\left[\frac{\lambda_{n}}{1-\gamma \rho}\left\|\gamma f\left(x_{n}\right)-\tilde{x}\right\|\left\|A x_{n}-A \tilde{x}\right\|\right. \\
& \left.+\frac{1-\alpha_{n}}{1-\gamma \rho}\left\langle\gamma f(\tilde{x})-\tilde{x}, x_{n}-\tilde{x}\right\rangle+\frac{\alpha_{n}}{2(1-\gamma \rho)}\left(\left\|\gamma f\left(x_{n}\right)-\tilde{x}\right\|^{2}+\left\|x_{n}-\tilde{x}\right\|^{2}\right)\right] .
\end{aligned}
$$

It is clear that $\sum_{n} 2 \kappa(1-\gamma \rho) \alpha_{n}=\infty$ and

$$
\begin{aligned}
& \limsup _{n \rightarrow \infty}\left\{\frac{\lambda_{n}}{1-\gamma \rho}\left\|\gamma f\left(x_{n}\right)-\tilde{x}\right\|\left\|A x_{n}-A \tilde{x}\right\|+\frac{1-\alpha_{n}}{1-\gamma \rho}\left\langle\gamma f(\tilde{x})-\tilde{x}, x_{n}-\tilde{x}\right\rangle\right. \\
& \left.+\frac{\alpha_{n}}{2(1-\gamma \rho)}\left(\left\|\gamma f\left(x_{n}\right)-\tilde{x}\right\|^{2}+\left\|x_{n}-\tilde{x}\right\|^{2}\right)\right\} \leq 0 .
\end{aligned}
$$

Therefore, we can apply Lemma 2.5 to conclude that $x_{n} \rightarrow \tilde{x}$. This completes the proof.

Remark 3.3 One quite often seeks a particular solution of a given nonlinear problem, in particular, the minimum-norm element. For instance, given a closed convex subset $C$ of a Hilbert space $H_{1}$ and a bounded linear operator $W: H_{1} \rightarrow H_{2}$, where $H_{2}$ is another Hilbert space. The $C$-constrained pseudoinverse of $W, W_{C}^{\dagger}$, is then defined as the minimum-norm solution of the constrained minimization problem

$$
W_{C}^{\dagger}(b):=\arg \min _{x \in C}\|W x-b\|
$$

which is equivalent to the fixed point problem

$$
u=\operatorname{proj}_{C}\left(u-\mu W^{*}(W u-b)\right)
$$

where $W^{*}$ is the adjoint of $W$ and $\mu>0$ is a constant, and $b \in H_{2}$ is such that $P_{\overline{W(C)}}(b) \in$ $W(C)$. From Theorems 3.1 and 3.2, we get the following corollaries which can find the minimum-norm element in $F(S) \cap(A+B)^{-1} 0$; that is, find $\tilde{x} \in F(S) \cap(A+B)^{-1} 0$ such that

$$
\tilde{x}=\arg \min _{x \in F(S) \cap(A+B)^{-1} 0}\|x\| .
$$

Corollary 3.4 Let $C$ be a nonempty closed convex subset of a real Hilbert space H. Let $A$ be an $\alpha$-inverse strongly monotone mapping from $C$ into $H$. Let $B$ be a maximal monotone operator on $H$ such that the domain of $B$ is included in C. Let $J_{\lambda}^{B}=(I+\lambda B)^{-1}$ be the resolvent of $B$ for any $\lambda>0$ and $S$ be a nonexpansive mapping from $C$ into itself such that $F(S) \cap(A+$ $B)^{-1} 0 \neq \emptyset$. Let $\lambda$ and $\kappa$ be two constants satisfying $a \leq \lambda \leq b$, where $[a, b] \subset(0,2 \alpha)$ and $\kappa \in(0,1)$. For any $t \in\left(0,1-\frac{\lambda}{2 \alpha}\right)$, let $\left\{x_{t}\right\} \subset C$ be a net generated by

$$
x_{t}=(1-\kappa) S x_{t}+\kappa J_{\lambda}^{B}\left((1-t) x_{t}-\lambda A x_{t}\right)
$$

Then the net $\left\{x_{t}\right\}$ converges strongly as $t \rightarrow 0+$ to a point $\tilde{x}=P_{F(S) \cap(A+B)^{-1} 0}(0)$ which is the minimum-norm element in $F(S) \cap(A+B)^{-1} 0$.

Corollary 3.5 Let $C$ be a closed convex subset of a real Hilbert space H. Let $A$ be an $\alpha$ inverse strongly monotone mapping from $C$ into $H$ and let $B$ be a maximal monotone operator on $H$ such that the domain of $B$ is included in C. Let $J_{\lambda}^{B}=(I+\lambda B)^{-1}$ be the resolvent 
of $B$ for any $\lambda>0$ such that $(A+B)^{-1} 0 \neq \emptyset$. Let $\lambda$ be a constant satisfying $a \leq \lambda \leq b$, where $[a, b] \subset(0,2 \alpha)$. For any $t \in\left(0,1-\frac{\lambda}{2 \alpha}\right)$, let $\left\{x_{t}\right\} \subset C$ be a net generated by

$$
x_{t}=J_{\lambda}^{B}\left((1-t) x_{t}-\lambda A x_{t}\right) .
$$

Then the net $\left\{x_{t}\right\}$ converges strongly as $t \rightarrow 0+$ to a point $\tilde{x}=P_{(A+B)^{-1} 0}(0)$, which is the minimum-norm element in $(A+B)^{-1} 0$.

Corollary 3.6 Let $C$ be a nonempty closed convex subset of a real Hilbert space H. Let A be an $\alpha$-inverse strongly monotone mapping from $C$ into $H$. Let $B$ be a maximal monotone operator on $H$ such that the domain of $B$ is included in C. Let $J_{\lambda}^{B}=(I+\lambda B)^{-1}$ be the resolvent of $B$ for any $\lambda>0$ and let $S$ be a nonexpansive mapping from $C$ into itself such that $F(S) \cap$ $(A+B)^{-1} 0 \neq \emptyset$. For any $x_{0} \in C$, let $\left\{x_{n}\right\} \subset C$ be a sequence generated by

$$
x_{n+1}=(1-\kappa) S x_{n}+\kappa J_{\lambda_{n}}^{B}\left(\left(1-\alpha_{n}\right) x_{n}-\lambda_{n} A x_{n}\right)
$$

for all $n \geq 0$, where $\kappa \in(0,1),\left\{\lambda_{n}\right\} \subset(0,2 \alpha)$, and $\left\{\alpha_{n}\right\} \subset(0,1)$ satisfy the following conditions:

(a) $\lim _{n \rightarrow \infty} \alpha_{n}=0, \lim _{n \rightarrow \infty} \frac{\alpha_{n+1}}{\alpha_{n}}=1$, and $\sum_{n} \alpha_{n}=\infty$;

(b) $a\left(1-\alpha_{n}\right) \leq \lambda_{n} \leq b\left(1-\alpha_{n}\right)$, where $[a, b] \subset(0,2 \alpha)$ and $\lim _{n \rightarrow \infty} \frac{\lambda_{n+1}-\lambda_{n}}{\alpha_{n}}=0$.

Then the sequence $\left\{x_{n}\right\}$ converges strongly to a point $\tilde{x}=P_{F(S) \cap(A+B)^{-1} 0}(0)$, which is the minimum-norm element in $F(S) \cap(A+B)^{-1} 0$.

Corollary 3.7 Let $C$ be a closed convex subset of a real Hilbert space H. Let $A$ be an $\alpha$ inverse strongly monotone mapping from $C$ into $H$ and let $B$ be a maximal monotone operator on $H$ such that the domain of $B$ is included in C. Let $J_{\lambda}^{B}=(I+\lambda B)^{-1}$ be the resolvent of $B$ for any $\lambda>0$ such that $(A+B)^{-1} 0 \neq \emptyset$. For any $x_{0} \in C$, let $\left\{x_{n}\right\} \subset C$ be a sequence generated by

$$
x_{n+1}=(1-\kappa) x_{n}+\kappa J_{\lambda_{n}}^{B}\left(\left(1-\alpha_{n}\right) x_{n}-\lambda_{n} A x_{n}\right)
$$

for all $n \geq 0$, where $\kappa \in(0,1),\left\{\lambda_{n}\right\} \subset(0,2 \alpha)$, and $\left\{\alpha_{n}\right\} \subset(0,1)$ satisfy the following conditions:

(a) $\lim _{n \rightarrow \infty} \alpha_{n}=0, \lim _{n \rightarrow \infty} \frac{\alpha_{n+1}}{\alpha_{n}}=1$, and $\sum_{n} \alpha_{n}=\infty$;

(b) $a\left(1-\alpha_{n}\right) \leq \lambda_{n} \leq b\left(1-\alpha_{n}\right)$, where $[a, b] \subset(0,2 \alpha)$ and $\lim _{n \rightarrow \infty} \frac{\lambda_{n+1}-\lambda_{n}}{\alpha_{n}}=0$.

Then the sequence $\left\{x_{n}\right\}$ converges strongly to a point $\tilde{x}=P_{(A+B)^{-1} 0}(0)$, which is the minimum-norm element in $(A+B)^{-1} 0$.

Remark 3.8 The present paper provides some methods which do not use projection for finding the minimum-norm solution problem.

\section{Applications}

Next, we consider the problem for finding the minimum-norm solution of a mathematical model related to equilibrium problems. Let $C$ be a nonempty closed convex subset of a Hilbert space and $G: C \times C \rightarrow R$ be a bifunction satisfying the following conditions:

(E1) $G(x, x)=0$ for all $x \in C$;

(E2) $G$ is monotone, i.e., $G(x, y)+G(y, x) \leq 0$ for all $x, y \in C$; 
(E3) for all $x, y, z \in C, \lim \sup _{t \downarrow 0} G(t z+(1-t) x, y) \leq G(x, y)$;

(E4) for all $x \in C, G(x, \cdot)$ is convex and lower semicontinuous.

Then the mathematical model related to the equilibrium problem (with respect to $C$ ) is as follows:

Find $\tilde{x} \in C$ such that

$$
G(\tilde{x}, y) \geq 0
$$

for all $y \in C$. The set of such solutions $\tilde{x}$ is denoted by $E P(G)$.

The following lemma appears implicitly in Blum and Oettli [36].

Lemma 4.1 Let $C$ be a nonempty closed convex subset of a Hilbert space $H$. Let $G$ be a bifunction from $C \times C$ into $R$ satisfying the conditions (E1)-(E4). Then, for any $r>0$ and $x \in H$, there exists $z \in C$ such that

$$
G(z, y)+\frac{1}{r}\langle y-z, z-x\rangle \geq 0
$$

for all $y \in C$.

The following lemma was given in Combettes and Hirstoaga [37].

Lemma 4.2 Assume that $G$ is a bifunction from $C \times C$ into $R$ satisfying the conditions (E1)-(E4). For any $r>0$ and $x \in H$, define a mapping $T_{r}: H \rightarrow C$ as follows:

$$
T_{r}(x)=\left\{z \in C: G(z, y)+\frac{1}{r}\langle y-z, z-x\rangle \geq 0, \forall y \in C\right\}
$$

for all $x \in H$. Then the following hold:

(a) $T_{r}$ is single-valued;

(b) $T_{r}$ is a firmly nonexpansive mapping, i.e., for all $x, y \in H$,

$$
\left\|T_{r} x-T_{r} y\right\|^{2} \leq\left\langle T_{r} x-T_{r} y, x-y\right\rangle
$$

(c) $F\left(T_{r}\right)=E P(G)$;

(d) $E P(G)$ is closed and convex.

We call such a $T_{r}$ the resolvent of $G$ for any $r>0$. Using Lemmas 4.1 and 4.2, we have the following lemma (see [38] for a more general result).

Lemma 4.3 Let $C$ be a nonempty closed convex subset of a Hilbert space $H$. Let $G$ be a bifunction from $C \times C$ into $R$ satisfying the conditions (E1)-(E4). Let $A_{G}$ be a multi-valued mapping from $H$ into itself defined by

$$
A_{G} \mathcal{X}= \begin{cases}\{z \in H: G(x, y) \geq\langle y-x, z\rangle, \forall y \in C\}, & x \in C, \\ \emptyset, & x \notin C .\end{cases}
$$


Then $\operatorname{EP}(G)=A_{G}^{-1}(0)$ and $A_{G}$ is a maximal monotone operator with $\operatorname{dom}\left(A_{G}\right) \subset C$. Further, for any $x \in H$ and $r>0$, the resolvent $T_{r}$ of $G$ coincides with the resolvent of $A_{G}$, i.e.,

$$
T_{r} x=\left(I+r A_{G}\right)^{-1} x .
$$

Form Lemma 4.3 and Theorems 3.1 and 3.2, we have the following results.

Theorem 4.4 Let $C$ be a nonempty closed convex subset of a real Hilbert space H. Let $G$ be a bifunction from $C \times C$ into $R$ satisfying the conditions (E1)-(E4) and $T_{r}$ be the resolvent of $G$ for any $r>0$. Let $S$ be a nonexpansive mapping from $C$ into itself such that $F(S) \cap E P(G) \neq \emptyset$. For any $t \in(0,1)$, let $\left\{x_{t}\right\} \subset C$ be a net generated by

$$
x_{t}=(1-\kappa) S x_{t}+\kappa T_{r}\left((1-t) x_{t}\right) .
$$

Then the net $\left\{x_{t}\right\}$ converges strongly as $t \rightarrow 0+$ to a point $\tilde{x}=P_{F(S) \cap E P(G)}(0)$, which is the minimum-norm element in $F(S) \cap E P(G)$.

Proof From Lemma 4.3, we know $A_{G}$ is maximal monotone. Thus, in Theorem 3.1, we can set $J_{\lambda}^{B}=T_{r}$. At the same time, in Theorem 3.1, we can choose $f=0$ and $A=0$, and (3.1) reduces to

$$
x_{t}=(1-\kappa) S x_{t}+\kappa T_{r}\left((1-t) x_{t}\right) .
$$

Consequently, from Theorem 3.1, we get the desired result. This completes the proof.

Corollary 4.5 Let C be a nonempty closed convex subset of a real Hilbert space H. Let G be a bifunction from $C \times C$ into $R$ satisfying the conditions (E1)-(E4) and $T_{r}$ be the resolvent of $G$ for any $r>0$. Suppose that $E P(G) \neq \emptyset$. For any $t \in(0,1)$, let $\left\{x_{t}\right\} \subset C$ be a net generated by

$$
x_{t}=T_{r}\left((1-t) x_{t}\right) .
$$

Then the net $\left\{x_{t}\right\}$ converges strongly as $t \rightarrow 0+$ to a point $\tilde{x}=P_{E P(G)}(0)$, which is the minimum-norm element in $E P(G)$.

Theorem 4.6 Let $C$ be a nonempty closed and convex subset of a real Hilbert space $H$. Let $G$ be a bifunction from $C \times C$ into $R$ satisfying the conditions (E1)-(E4) and $T_{\lambda}$ be the resolvent of $G$ for any $\lambda>0$. Let $S$ be a nonexpansive mapping from $C$ into itself such that $F(S) \cap E P(G) \neq \emptyset$. For any $x_{0} \in C$, let $\left\{x_{n}\right\} \subset C$ be a sequence generated by

$$
x_{n+1}=(1-\kappa) S x_{n}+\kappa T_{\lambda_{n}}\left(\left(1-\alpha_{n}\right) x_{n}\right)
$$

for all $n \geq 0$, where $\kappa \in(0,1),\left\{\lambda_{n}\right\} \subset(0, \infty)$, and $\left\{\alpha_{n}\right\} \subset(0,1)$ satisfy the conditions:

(a) $\lim _{n \rightarrow \infty} \alpha_{n}=0, \lim _{n \rightarrow \infty} \frac{\alpha_{n+1}}{\alpha_{n}}=1$, and $\sum_{n} \alpha_{n}=\infty$;

(b) $a \leq \lambda_{n} \leq b$, where $[a, b] \subset(0, \infty)$ and $\lim _{n \rightarrow \infty} \frac{\lambda_{n+1}-\lambda_{n}}{\alpha_{n}}=0$.

Then the sequence $\left\{x_{n}\right\}$ converges strongly to a point $\tilde{x}=P_{F(S) \cap E P(G)}(0)$, which is the minimum-norm element in $F(S) \cap E P(G)$. 
Proof From Lemma 4.3, we know $A_{G}$ is maximal monotone. Thus, in Theorem 3.2, we can set $J_{\lambda_{n}}^{B}=T_{\lambda_{n}}$. At the same time, in Theorem 3.2, we can choose $f=0$ and $A=0$, and (3.10) reduces to

$$
x_{n+1}=(1-\kappa) S x_{n}+\kappa T_{\lambda_{n}}\left(\left(1-\alpha_{n}\right) x_{n}\right)
$$

for all $n \geq 0$. Consequently, from Theorem 3.2, we get the desired result. This completes the proof.

Corollary 4.7 Let C be a nonempty closed convex subset of a real Hilbert space H. Let G be a bifunction from $C \times C$ into $R$ satisfying the conditions (E1)-(E4) and $T_{\lambda}$ be the resolvent of $G$ for any $\lambda>0$. Suppose $E P(G) \neq \emptyset$. For any $x_{0} \in C$, let $\left\{x_{n}\right\} \subset C$ be a sequence generated by

$$
x_{n+1}=(1-\kappa) x_{n}+\left(1-\beta_{n}\right) T_{\lambda_{n}}\left(\left(1-\alpha_{n}\right) x_{n}\right)
$$

for all $n \geq 0$, where $\kappa \in(0,1),\left\{\lambda_{n}\right\} \subset(0, \infty)$, and $\left\{\alpha_{n}\right\} \subset(0,1)$ satisfy the following conditions:

(a) $\lim _{n \rightarrow \infty} \alpha_{n}=0, \lim _{n \rightarrow \infty} \frac{\alpha_{n+1}}{\alpha_{n}}=1$, and $\sum_{n} \alpha_{n}=\infty$;

(b) $a \leq \lambda_{n} \leq b$, where $[a, b] \subset(0, \infty)$ and $\lim _{n \rightarrow \infty} \frac{\lambda_{n+1}-\lambda_{n}}{\alpha_{n}}=0$.

Then the sequence $\left\{x_{n}\right\}$ converges strongly to a point $\tilde{x}=P_{E P(G)}(0)$, which is the minimumnorm element in $E P(G)$.

\section{Competing interests}

The authors declare that they have no competing interests.

\section{Authors' contributions}

All authors contributed equally and significantly in writing this article. All authors read and approved the final manuscript.

\section{Author details}

${ }^{1}$ Department of Mathematics, King Abdulaziz University, Jeddah, 21589, Saudi Arabia. ${ }^{2}$ Department of Mathematics Education and the RINS, Gyeongsang National University, Chinju, 660-701, Korea. ${ }^{3}$ Department of Mathematics, Tianjin Polytechnic University, Tianjin, 300387, China. ${ }^{4}$ School of Mathematics and Information Science, Beifang University of Nationalities, Yinchuan, 750021, China.

\section{Acknowledgements}

This project was funded by the Deanship of Scientific Research (DSR), King Abdulaziz University, under Grant No. (31-130-35-HiCi) and the fifth author was supported in part by NNSF of China (61362033) and NGY2012097.

Received: 19 March 2014 Accepted: 27 June 2014 Published: 18 Aug 2014

\section{References}

1. Lu, X, Xu, HK, Yin, X: Hybrid methods for a class of monotone variational inequalities. Nonlinear Anal. 71, 1032-1041 (2009)

2. Rockafella, RT: Monotone operators and the proximal point algorithm. SIAM J. Control Optim. 14, $877-898$ (1976)

3. Moudafi, A, Noor, MA: Sensitivity analysis of variational inclusions by the Wiener-Hopf equations technique. J. Appl. Math. Stoch. Anal. 12, 223-232 (1999)

4. Dong, H, Lee, BS, Huang, NJ: Sensitivity analysis for generalized parametric implicit quasi-variational inequalities. Nonlinear Anal. Forum 6, 313-320 (2001)

5. Agarwal, RP, Huang, NJ, Tan, MY: Sensitivity analysis for a new system of generalized nonlinear mixed quasi-variational inclusions. Appl. Math. Lett. 17, 345-352 (2004)

6. Jeong, JU: A system of parametric generalized nonlinear mixed quasi-variational inclusions in Lp spaces. J. Appl. Math. Comput. 19, 493-506 (2005)

7. Lan, HY: A stable iteration procedure for relaxed cocoercive variational inclusion systems based on $(A, \eta)$-monotone operators. J. Comput. Anal. Appl. 9, 147-157 (2007)

8. Zhang, SS, Lee Joseph, HW, Chan, CK: Algorithms of common solutions for quasi variational inclusion and fixed point problems. Appl. Math. Mech. 29, 571-581 (2008) 
9. Peng, JW, Wang, Y, Shyu, DS, Yao, JC: Common solutions of an iterative scheme for variational inclusions, equilibrium problems, and fixed point problems. J. Inequal. Appl. 2008, Article ID 720371 (2008)

10. Ansari, QH, Balooee, J, Yao, J-C: Iterative algorithms for systems of extended regularized nonconvex variational inequalities and fixed point problems. Appl. Anal. 93, 972-993 (2014)

11. Bauschke, HH, Combettes, PL: A Dykstra-like algorithm for two monotone operators. Pac. J. Optim. 4, 383-391 (2008)

12. Bauschke, $\mathrm{HH}$, Combettes, $\mathrm{PL}$, Reich, $\mathrm{S}$ : The asymptotic behavior of the composition of two resolvents. Nonlinear Anal. 60, 283-301 (2005)

13. Ceng, L-C, Al-Otaibi, A, Ansari, QH, Latif, A: Relaxed and composite viscosity methods for variational inequalities, fixed points of nonexpansive mappings and zeros of accretive operators. Fixed Point Theory Appl. 2014, 29 (2014)

14. Ceng, L-C, Ansari, QH, Schaible, S, Yao, J-C: Iterative methods for generalized equilibrium problems, systems of general generalized equilibrium problems and fixed point problems for nonexpansive mappings in Hilbert spaces. Fixed Point Theory 12, 293-308 (2011)

15. Combettes, PL: Solving monotone inclusions via compositions of nonexpansive averaged operators. Optimization 53, 475-504 (2004)

16. Combettes, PL, Hirstoaga, SA: Approximating curves for nonexpansive and monotone operators. J. Convex Anal. 13 633-646 (2006)

17. Combettes, PL, Hirstoaga, SA: Visco-penalization of the sum of two monotone operators. Nonlinear Anal. 69, 579-591 (2008)

18. Eckstein, J, Bertsekas, DP: On the Douglas-Rachford splitting method and the proximal point algorithm for maximal monotone operators. Math. Program. 55, 293-318 (1992)

19. Fang, YP, Huang, NJ: H-Monotone operator resolvent operator technique for quasi-variational inclusions. Appl. Math Comput. 145, 795-803 (2003)

20. Lin, LJ: Variational inclusions problems with applications to Ekeland's variational principle, fixed point and optimization problems. J. Glob. Optim. 39, 509-527 (2007)

21. Lions, P-L, Mercier, B: Splitting algorithms for the sum of two nonlinear operators. SIAM J. Numer. Anal. 16, 964-979 (1979)

22. Moudafi, A: On the regularization of the sum of two maximal monotone operators. Nonlinear Anal. 42, 1203-1208 (2009)

23. Passty, GB: Ergodic convergence to a zero of the sum of monotone operators in Hilbert spaces. J. Math. Anal. Appl. 72, 383-390 (1979)

24. Takahashi, S, Takahashi, W, Toyoda, M: Strong convergence theorems for maximal monotone operators with nonlinear mappings in Hilbert spaces. J. Optim. Theory Appl. 147, 27-41 (2010)

25. Ding, F, Chen, T: Iterative least squares solutions of coupled Sylvester matrix equations. Syst. Control Lett. 54, 95-107 (2005)

26. Liu, X, Cui, Y: Common minimal-norm fixed point of a finite family of nonexpansive mappings. Nonlinear Anal. 73 , 76-83 (2010)

27. Sabharwal, A, Potter, LC: Convexly constrained linear inverse problems: iterative least-squares and regularization. IEEE Trans. Signal Process. 46, 2345-2352 (1998)

28. Yao, Y, Chen, R, Xu, HK: Schemes for finding minimum-norm solutions of variational inequalities. Nonlinear Anal. 72 3447-3456 (2010)

29. Yao, Y, Liou, YC: Composite algorithms for minimization over the solutions of equilibrium problems and fixed point problems. Abstr. Appl. Anal. 2010, Article ID 763506 (2010)

30. Bauschke, HH, Borwein, JM: On projection algorithms for solving convex feasibility problems. SIAM Rev. 38, 367-426 (1996)

31. Censor, Y, Zenios, SA: Parallel Optimization: Theory, Algorithms and Applications. Oxford University Press, New York (1997)

32. Takahashi, W, Toyoda, M: Weak convergence theorems for nonexpansive mappings and monotone mappings. J. Optim. Theory Appl. 118, 417-428 (2003)

33. Goebel, K, Kirk, WA: Topics in Metric Fixed Point Theory. Cambridge Studies in Advanced Mathematics, vol. 28 Cambridge University Press, Cambridge (1990)

34. Suzuki, T: Strong convergence theorems for infinite families of nonexpansive mappings in general Banach spaces. Fixed Point Theory Appl. 2005, 103-123 (2005)

35. Xu, HK: Iterative algorithms for nonlinear operators. J. Lond. Math. Soc. 2, 1-17 (2002)

36. Blum, E, Oettli, W: From optimization and variational inequalities to equilibrium problems. Math. Stud. 63, 123-145 (1994)

37. Combettes, PL, Hirstoaga, SA: Equilibrium programming in Hilbert spaces. J. Nonlinear Convex Anal. 6, 117-136 (2005)

38. Aoyama, K, Kimura, Y, Takahashi, W, Toyoda, M: On a strongly nonexpansive sequence in Hilbert spaces. J. Nonlinear Convex Anal. 8, 471-489 (2007)

10.1186/1687-1812-2014-174

Cite this article as: Abdou et al.: Parallel algorithms for variational inclusions and fixed points with applications. Fixed Point Theory and Applications 2014, 2014:174 